\title{
Dust observations with antenna measurements and its prospects for observations with Parker Solar Probe and Solar Orbiter
}

\author{
Ingrid Mann ${ }^{1}$, Libor Nouzák $^{2}$, Jakub Vaverka ${ }^{2}$, Tarjei Antonsen ${ }^{1}$, Åshild Fredriksen ${ }^{1}$, Karine Issautier $^{3}$, \\ David Malaspina $^{4}$, Nicole Meyer-Vernet ${ }^{3}$, Jiří Pavlü ${ }^{2}$, Zoltan Sternovsky ${ }^{4}$, Joan Stude ${ }^{5}$, Shengyi Ye ${ }^{6,7}$, and \\ Arnaud Zaslavsky ${ }^{3}$ \\ ${ }^{1}$ Department of Physics and Technology, UiT The Arctic University of Norway, 9037, Troms $\varnothing$, Norway \\ ${ }^{2}$ Department of Surface and Plasma Science, Charles University Prague, 18000 Prague, Czech Republic \\ ${ }^{3}$ LESIA - Observatoire de Paris, Université PSL, CNRS, Sorbonne Université, Université de Paris, \\ 5 place Jules Janssen, 92195 Meudon, France \\ ${ }^{4}$ Laboratory for Atmospheric and Space Physics, University of Colorado, Boulder, CO 80303, USA \\ ${ }^{5}$ Deutsches Zentrum für Luft- und Raumfahrt, Institut für Physik der Atmosphäre, Oberpfaffenhofen, Germany \\ ${ }^{6}$ Department of Physics and Astronomy, University of Iowa, Iowa City, 52242-1479, Iowa, USA \\ ${ }^{7}$ Department of Earth and Space Sciences, Southern University of Science and Technology, Shenzhen, China
}

Correspondence: Ingrid Mann (ingrid.b.mann@uit.no)

Received: 28 June 2019 - Discussion started: 15 July 2019

Accepted: 7 October 2019 - Published: 10 December 2019

\begin{abstract}
The electric and magnetic field instrument suite FIELDS on board the NASA Parker Solar Probe and the radio and plasma waves instrument RPW on the ESA Solar Orbiter mission that explore the inner heliosphere are sensitive to signals generated by dust impacts. Dust impacts have been observed using electric field antennas on spacecraft since the 1980s and the method was recently used with a number of space missions to derive dust fluxes. Here, we consider the details of dust impacts, subsequent development of the impact generated plasma and how it produces the measured signals. We describe empirical approaches to characterise the signals and compare these in a qualitative discussion of laboratory simulations to predict signal shapes for spacecraft measurements in the inner solar system. While the amount of charge production from a dust impact will be higher near the Sun than observed in the interplanetary medium before, the amplitude of pulses is determined by the recovery behaviour that is different near the Sun since it varies with the plasma environment.
\end{abstract}

\section{Introduction}

The space missions Parker Solar Probe and Solar Orbiter to explore the inner heliosphere and close vicinity of the Sun carry antenna experiments that respond to dust impacts on the spacecraft. Parker Solar Probe (Parker Probe) is a NASA mission that was launched in August 2018 and collects data in the vicinity of the Sun, $\sim 10$ solar radii from the solar equator at the closest distance. The mission payload includes the electric and magnetic field instrument suite FIELDS (Bale et al., 2016). Solar Orbiter is an ESA mission with a launch planned in 2020 (Mueller and al., 2019). It will study the vicinity of the Sun as close as $0.3 \mathrm{AU}$ and at maximum $35^{\circ}$ inclination from the solar equatorial plane and includes the Radio and Plasma Waves (RPW) experiment (Maksimovic et al., 2019). Dust impacts are observed with electric antennas for field measurements since the 1980s, beginning with the Voyager mission (see Gurnett et al., 1997b, Meyer-Vernet, 2001). The method was recently used with a number of space missions to derive dust fluxes. While antenna measurements do not replace those of dedicated dust detectors, they are interesting because many space missions carry electric field instruments. In addition, antenna measurements can observe lower dust fluxes because of their large collecting area of the whole spacecraft in comparison to the 
small collecting area of dust detectors. A limitation of the antenna measurements is that they do not provide information on dust composition and only limited information, if any, on impact direction and dust mass. These derived values are highly uncertain (Meyer-Vernet et al., 2009; Zaslavsky et al., 2012; Malaspina et al., 2015). The relationship between dust impacts and the signals they produce in electric field instruments has also been considered in new instrument development and laboratory measurements.

Cosmic dust particles are one of the major constituents of the interplanetary medium in the inner heliosphere, and knowledge on dust near the Sun has so far been based on remote observations and model assumptions. An exception are the measurements of the HELIOS mission, with two spacecraft that reached a minimum distance $0.31 \mathrm{AU}$ from the Sun and each carried a dust detector (Grün et al., 1980). Our basic understanding (see for example Mann et al., 2004) is that large (> micrometre) dust particles that are fragments of comets and asteroids are in Keplerian orbits around the Sun. Their velocities and number densities increase with decreasing distance from the Sun. Fragments are produced in dustdust collisions for which the rates increase with decreasing distance from the Sun. The majority of fragments smaller than a micrometre are pushed outward by radiation pressure (Wehry, and Mann, 1999) and deflected byelectro-magnetic forces (Mann et al., 2004; Czechowski and Mann, 2010). In addition to the interplanetary dust, interstellar dust particles stream into the inner heliosphere from an interstellar upstream direction and move roughly parallel to the ecliptic plane (Mann, 2010). Because of repulsion by the radiation pressure force, only the large interstellar dust reaches the inner heliosphere. A large fraction of dust is destroyed in the inner heliosphere, in sublimation and other destruction processes, and this generates a dust-free zone. Sun-grazing comets (cf. Jones et al., 2018) are a local highly variable source for dust particles (see Fig. 1). Dust material is released in the ambient solar wind, a process which is not well quantified. The solid fragments that are not fully destroyed are pushed outward and produce the small-sized portion of the interplanetary dust flux observed near Earth's orbit. Parker Probe and Solar Orbiter will for the first time explore the inner heliosphere in situ. The dust impacts on the spacecraft will influence electric field measurements on these spacecraft and provide an opportunity to study the dust environments of the inner heliosphere.

This paper discusses dust detection with electric field measurements based on recent observations from several spacecraft, and it discusses the prospects of studying dust impacts with the two space missions to the inner heliosphere. We start by describing the impact ionisation of dust particles (Sect. 2), followed by a qualitative discussion of the dust impact signal generation in the vicinity of the spacecraft (Sect. 3) and resulting antenna signal shapes (Sect. 4). Section 5 discusses the signals and observations made recently with other space missions. In Sect. 6 we describe our current state of knowl- edge on dust in the inner heliosphere and in Sect. 7 we discuss implications for observations with Parker Probe and Solar Orbiter.

\section{Dust impact ionisation}

Dust impacts on the spacecraft body generate clouds of ions and electrons through a process known as impact ionisation. An impact ionisation model suggested by Drapatz and Michel (1974) can be summarised as follows: Dust grains impacting onto the spacecraft at speeds that are supersonic in the target solid, i.e. faster than a few kilometres per second, produce a shock compression wave in the solid which shatters, vaporises and ionises the dust as well as the material of the target, where an impact crater forms. Free charges in the form of electrons and atomic and molecular ions are produced by thermal ionisation of the impact vapour. The initial ionisation is followed by recombination and thermalisation and a residual ionisation remains in the impact vapour. The amount of the residual ionisation can be derived from laboratory measurements of the charge production. An empirical relation describes the charge production $Q$, as a function of the dust mass $m$ and speed $v$ according to $Q=\xi m^{\alpha} v^{\beta}$, where $Q$ is given in Coulombs, the $m$ in kilograms and $v$ in kilometres per second. The exponents $\alpha$ and $\beta$ are dimensionless and determined from experimental data. The constant $\xi$ gives the proportionality and, as parameters $\alpha$ and $\beta$, it is dependent on both impactor and target composition. This impact ionisation model (see Drapatz and Michel, 1974) is in a good agreement with laboratory experiments in relatively thin targets, for speeds between the supersonic limit and some tens of kilometres per second. The model is based on the assumption that neutral vapour forms in the impact process and that it subsequently ionises because of its high temperature. Ionisation can also occur directly in the target where the propagating shock wave leads to a high-pressure high-temperature state so that the ejected target material is already ionised (see Hornung and Kissel, 1994). The properties of the vapour cloud are also depending on material composition and on the impact angle (Schultz et al., 2006). Moreover, the shock wave propagation in the target (spacecraft) and dust material produces not only vapour, but also solid fragments, and the fragment formation is predominant at smaller impact velocities (Jones et al., 1996). In summary, there is no theory that fully describes the charge production, sometimes also denoted as plasma production, that is caused by dust impacts on spacecraft. The following discussion uses the term impact cloud for the impact-generated electrons, ions and neutrals to avoid confusion with the surrounding plasma.

Charge production is often determined empirically in dust accelerator facilities, in recent years at those of the University of Stuttgart (Mocker et al., 2011) and the University of Colorado (Shu et al., 2012) for the range of impact ve- 


\section{Dust cloud near sun}

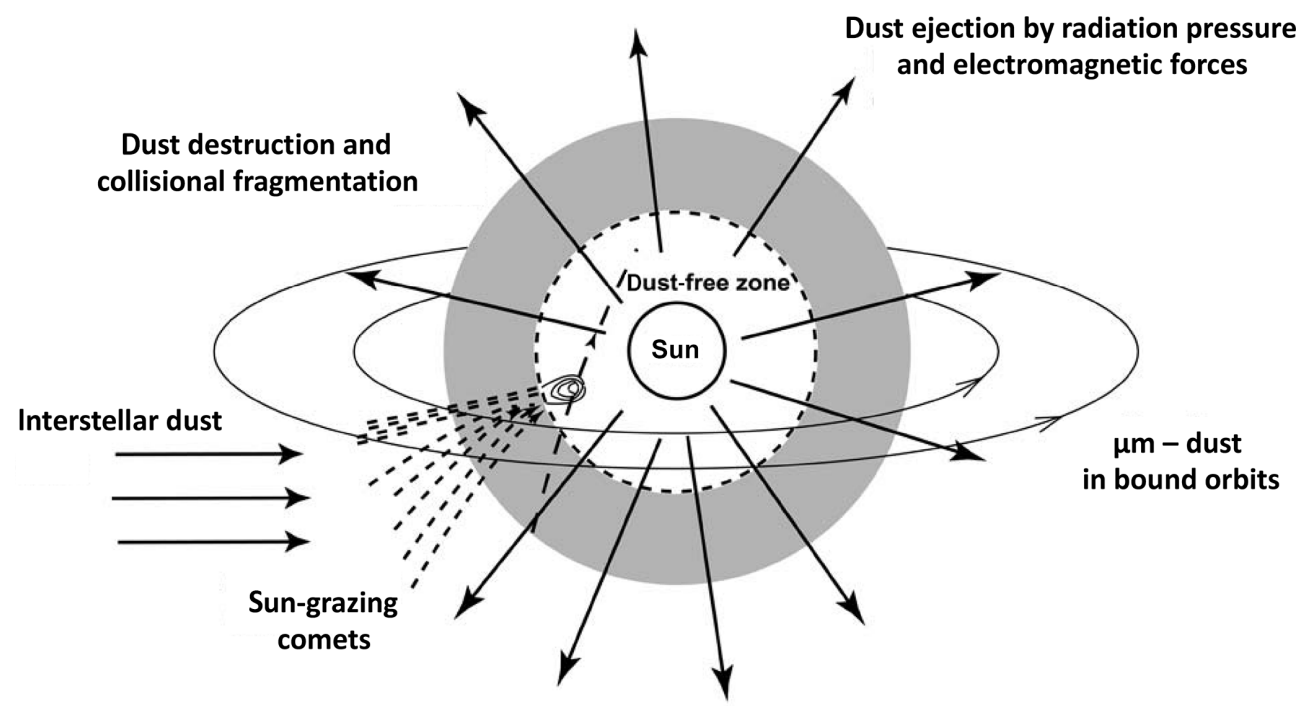

Figure 1. Sketch of different dust components and dust interactions in the vicinity from the Sun as given in an overview (adapted from Mann et al., 2014). Recent results are presented in Sect. 6.

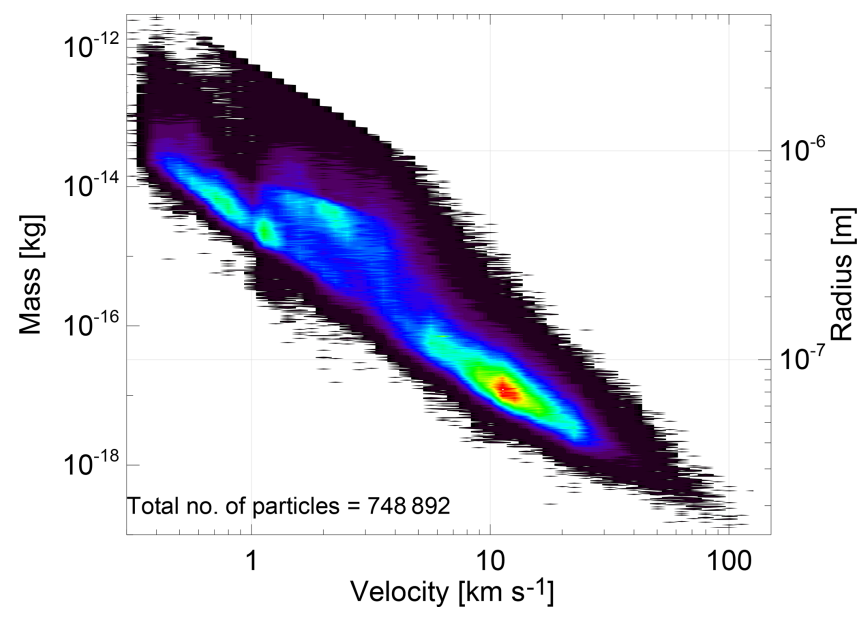

Figure 2. The mass vs. velocity distribution of iron dust particles generated by the accelerator facility at the University of Colorado.

locities shown in Fig. 2. The parameters to describe impact charge production derived from observations vary strongly for different impact materials; $\alpha$ has reported values between 0.7 and 1 while $\beta$ has been measured between 2.5 and 6.2 (see for example Dietzel et al., 1973; Auer, 2001; Collette et al., 2014, and references therein). An often-used relationship for dust impacts on spacecraft is $Q \approx 0.7 \mathrm{mv}^{3.5}$, which was reported for aluminium targets (McBride and McDonnell, 1999). It should be noted that the exponents also change for low impact energies (speeds below $\sim 1 \mathrm{~km} \mathrm{~s}^{-1}$ and sizes below $\sim 10 \mathrm{~nm}$ ). For low energy collisions, where fragments of significant sizes compared to the initial im- pactor survive, there may be surface effects such as capacitive contact charging (see for example John et al., 1980). The exponent also changes at high impact energies for speeds above $\sim 50 \mathrm{~km} \mathrm{~s}^{-1}$ and dust sizes above $\sim 1 \mu \mathrm{m}$ (Auer, 2001; Göller and Grün, 1989). Collette et al. (2014) measured the charge impact yield as a function of impact velocity for common materials used on spacecraft, and they point out the need for dedicated studies for impacts $>50 \mathrm{~km} \mathrm{~s}^{-1}$. For very large impact energies, where the impactor gets completely vaporised, surface effects are negligible and the charge generation can be modelled through hydrodynamic theory (Hornung and Kissel, 1994). Moreover, there is a dependence of impact angle on the charge generation (Schulz and Sugita, 2006; Collette et al., 2014). Based on spectroscopic analysis of 4.7$5.6 \mathrm{~km} \mathrm{~s}^{-1}$ impact flashes Sugita et al. (1998) find temperatures of about 4000 to $5000 \mathrm{~K}$ for the impact vapour cloud. Subsequent observations yield temperatures of 0.9 to $3 \mathrm{eV}$ for impact speeds varying from 10 to $40 \mathrm{~km} \mathrm{~s}^{-1}$ (Miyachi et al., 2008). Laboratory measurements find impact vapour ion temperatures of about $5 \mathrm{eV}$ at $4 \mathrm{~km} \mathrm{~s}^{-1}$ impact speed and $>10 \mathrm{eV}$ at $20 \mathrm{~km} \mathrm{~s}^{-1}$ (Collette et al., 2016).

\section{The impact process}

Formation of the dust impact signal involves the dust impact process, the interaction of the impact cloud with the surrounding plasma and finally the detection by electric field measurement. At the most basic level, dust impacts on the spacecraft body generate clouds of free electrons and positive or negative ions. These charged particles are attracted 
(a) SC negative

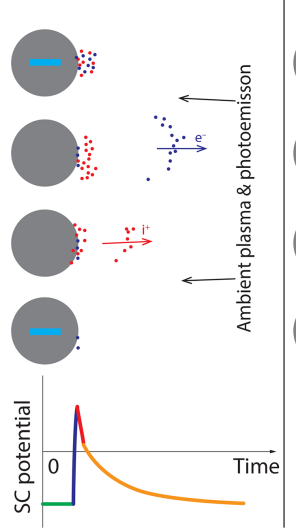

(b) $\mathrm{SC} \approx$ zero bias

(c) SC positive

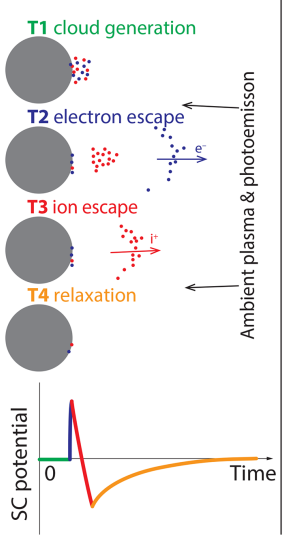

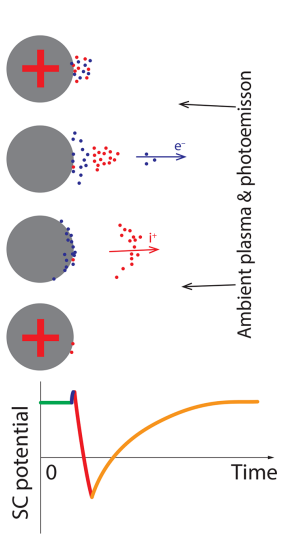

Figure 3. This figure sketches the impact process for a spacecraft that is slightly negatively charged (a), zero biased (b) and slightly positively charged (c). It is further described in the text and parameters given in Table 1.

to, or repulsed from, the spacecraft surface according to its electric potential relative to the surrounding ambient plasma. Charged particles from the impact cloud can be re-collected by the spacecraft or escape to free space and generate a transient deviation from the equilibrium spacecraft surface potential. The potential change can be positive or negative, e.g. escaping electrons generate a positive signal. Electrons are significantly faster than ions, and thus the signal generated by escaping electrons appears before the signal generated by escaping ions for the case when the spacecraft potential is not too large. The amplitude of the spacecraft potential deviation is given by the amount of escaped charges and by spacecraft capacitance. The spacecraft potential relaxes back to the equilibrium value via interaction with ambient plasma according to $\Phi_{\mathrm{SC}} \sim e^{-t / \tau}$, where $\tau$ is a characteristic relaxation time (Meyer-Vernet, 1985).

The different phases of the impact process for various spacecraft potentials (slightly positive, zero and slightly negative) are illustrated in Fig. 3. In the first phase (T1), at which the spacecraft is assumed to be in equilibrium potential, the impact occurs, and an impact cloud is generated (green). Some of the cloud particles may be re-collected. The second phase (T2) is characterised by electron escape and partial recollection depending on the target's potential, yielding an initial rise in signal strength (blue). The third phase (T3) is characterised by the ion escape, decreasing the spacecraft potential (red). The final phase (T4) is the relaxation phase when the spacecraft potential returns to the equilibrium value (orange). Individual time steps are summarised in Table 1 and sketched in Fig. 3. The ratio between escaping electrons and ions in phases T2 and T3 depend on the spacecraft potential. For example, more electrons than ions leave for a negatively charged spacecraft (Fig. 3a).

\subsection{Impact cloud generation and expansion - T1}

Charged particles at a small distance from the spacecraft body still influence its potential. The change in the spacecraft potential can thus not be observed directly after impact cloud formation but when charged particles are recollected or escape far enough and/or are sufficiently shielded by the ambient plasma or photoelectrons that their influence on the spacecraft potential is reduced (Meyer-Vernet et al., 2017). The number of escaping particles depends on initial impactor energy and velocity after the initial cloud expansion. It is possible to assume that impact cloud electrons move in random directions due to collisions with ions. This implies that half of the electrons move towards the spacecraft before they are influenced by spacecraft potential, whereas the other half moving initially outwards is recollected if the target potential is positive and higher than their temperature (in electronvolts). An alternative model assumes approximately half of both the electrons and the ions move towards the spacecraft. This model is in good agreement with recent laboratory measurements (Nouzák et al., 2018, see below). It is based on the assumption that the impact vapour is initially neutral and that the free charges form as a result of thermal ionisation in the impact vapour.

\subsection{Electron escape - T2}

The first part of the signal shown in Fig. 3 is generated by electrons escaping from the spacecraft body. The amplitude of the electron signal is reduced when the spacecraft is charged positively because some electrons are attracted back to the spacecraft. All electrons are re-collected when the positive spacecraft potential is significantly higher than the temperature of electrons (no electron part in the signal). This is a very fast process ( $\mu \mathrm{s})$ and the characteristic time depends on a number of parameters. Independent from the ambient plasma this process is influenced by the geometry of the system and specifics of the antenna and parts of the spacecraft body as well as the energy (velocity) of the electrons. The cloud expansion and internal shielding depends on the size of cloud formed by the impact. In space, the ambient plasma Debye length and magnitude of the photoemission current from the spacecraft determine the length scale of the spacecraft potential influence on the expansion.

\subsection{Ion escape - T3}

The ion escape works in a similar manner to electron escape, but happens at a lower rate if electron and ion temperature are comparable. The potential induced by ions on the spacecraft and antennas is progressively shielded by the ambient plasma electrons and photoelectrons. Moreover, escaping electrons can drag some of the ions behind them. This can result in double population of escaping ions: fast and slow. All ions 
Table 1. Explanation of impact process signal shape illustrated in Fig. 3.

\begin{tabular}{|c|c|c|c|c|}
\hline & (T1) Cloud generation & (T2) Electron escape & (T3) Ion escape & (T4) Relaxation \\
\hline $\begin{array}{l}\text { Negative spacecraft } \\
\text { potential }\left(U_{\mathrm{sc}, 0}<0\right)\end{array}$ & $\begin{array}{l}\text { Cloud generation } \\
\text { and expansion } \\
\left(U_{\mathrm{sc}, 0}<0\right)\end{array}$ & $\begin{array}{l}\text { Electron escape } \\
\left(U_{\mathrm{sc}, \mathrm{T} 2}>U_{\mathrm{sc}, 0}<0\right)\end{array}$ & $\begin{array}{l}\text { Ion escape suppressed } \\
\left(U_{\mathrm{sc}, \mathrm{T} 3}<U_{\mathrm{sc}}, \mathrm{T} 2\right)\end{array}$ & $\begin{array}{l}\text { Relaxation } \\
\left(U_{\mathrm{sc}, \mathrm{T} 4}=>U_{\mathrm{sc}, 0}\right)\end{array}$ \\
\hline $\begin{array}{l}\text { Spacecraft potential } \\
\text { zero }\left(U_{\mathrm{sc}, 0} \sim 0\right)\end{array}$ & $\begin{array}{l}\text { Cloud generation } \\
\text { and expansion } \\
\left(U_{\mathrm{sc}, 0}=0\right)\end{array}$ & $\begin{array}{l}\text { Partial electron escape } \\
\left(U_{\mathrm{sc}, \mathrm{T} 2}>U_{\mathrm{sc}, 0}=0\right)\end{array}$ & $\begin{array}{l}\text { Partial ion escape } \\
\left(U_{\mathrm{sc}, \mathrm{T} 3}<U_{\mathrm{sc}, \mathrm{T} 2}\right)\end{array}$ & $\begin{array}{l}\text { Relaxation } \\
\left(U_{\mathrm{sc}, \mathrm{T} 4}=>U_{\mathrm{sc}, 0}\right)\end{array}$ \\
\hline $\begin{array}{l}\text { Positive spacecraft } \\
\text { potential }\left(U_{\mathrm{sc}, 0}>0\right)\end{array}$ & $\begin{array}{l}\text { Cloud generation } \\
\text { and expansion } \\
\left(U_{\mathrm{sc}, 0}>0\right)\end{array}$ & $\begin{array}{l}\text { Electron escape } \\
\text { suppressed } \\
\left(U_{\mathrm{sc}, \mathrm{T} 2}>U_{\mathrm{sc}, 0}>0\right)\end{array}$ & $\begin{array}{l}\text { Ion escape } \\
\left(U_{\mathrm{sc}, \mathrm{T} 3}<U_{\mathrm{sc}, \mathrm{T} 2}\right)\end{array}$ & $\begin{array}{l}\text { Relaxation } \\
\left(U_{\mathrm{sc}, \mathrm{T} 4}=>U_{\mathrm{sc}, 0}\right)\end{array}$ \\
\hline
\end{tabular}

are recollected when the negative spacecraft potential is significantly higher than the temperature of ions.

\subsection{Relaxation - T4}

The spacecraft potential returns back to the equilibrium value due to interaction with ambient plasma. The relaxation time is determined by the ambient environment (plasma density, temperature, photoemission), and by the capacitance of the spacecraft and antennas. On the other hand, higher plasma density and stronger photoemission result in stronger currents from ambient plasma and thus in a significantly shorter relaxation time. The typical relaxation time measured by various spacecraft is in the range from $\approx 100 \mu$ up to several milliseconds. The relaxation time could be comparable or shorter than the ion escape or shielding time in dense plasma environments (or under strong photoemission), and by the capacitance of the spacecraft and antennas. This will result in a reduction of the detected signal and lowers the sensitivity of dust detection via electric field antennas. Relaxation time can also be reduced by active experiments, for example by ASPOC (active spacecraft potential control) (Vaverka et al., 2017b).

\section{Antenna signal shapes}

Electric field antennas can be operated as a dipole, where the voltage difference between two antenna booms is measured, or a monopole, where the voltage difference between an antenna boom and the spacecraft body is measured. It has been noted that the power spectral density of dust impact signals measured by monopole antennas is significantly larger than that measured by the dipole antennas (Meyer-Vernet, 1985; Tsintikidis et al., 1994; Meyer-Vernet et al., 2014), and this difference is attributed to the low sensitivity of a symmetric dipole antenna to dust impacts on spacecraft body. It is important to note that dust impact on a spacecraft body described by this model can be detected by the monopole electric field antenna as a potential drop between the spacecraft body and one antenna. A dipole configuration measur- ing electric field as a potential difference between two antennas can be utilised to detect a signal only when escaping electrons or ions influence the potential of one of the dipole antennas asymmetrically. The described model shows a strong dependence on the spacecraft potential. This can be compared with laboratory experiments for various polarities and sizes of bias voltage. A series of such measurement campaigns have been performed at the dust accelerator facility at the University of Colorado in order to aid the interpretation of signals collected in space. Collette et al. (2015) successfully identified different mechanisms of voltage signal generation on the antennas. The experiments performed by Nouzák et al. (2018) used a scale model of the Cassini spacecraft to investigate the differences between antennas operated in monopole vs. dipole modes. The results show that in the dipole mode the antennas are greatly insensitive to dust impacts occurring on the spacecraft and only impacts on the antennas generate clear signals. This study helped clarify the appropriate cross section to be used for calculating the density of dust populations encountered by the spacecraft (Ye et al., 2016b).

A few cases of impact events are shown in Fig. 4, derived from laboratory studies on a scaled-down Cassini model (Nouzák et al., 2018). Although the signals are measured in dipole configuration, since the dust impacts one of the dipole antennas, this configuration corresponds to monopole measurement when dust impacts the spacecraft body as described above. The laboratory experiment is performed in the vacuum chamber without ambient plasma. The relaxation process is simulated by discharge of the electronics circuit inside of the Cassini model.

Figure 4 shows different signal shapes measured in the Cassini laboratory simulation, and the signal development of the different stages are described for each case in Table 2:

- The signal shown in panel (a) is for a strongly negatively biased target potential. All electrons are repulsed from the spacecraft and all ions are recollected back to a strongly negatively biased target. The ion escape part 
(a) $U_{-}$

Dipole signals produced due to impact on dipole antenna $E_{u}$

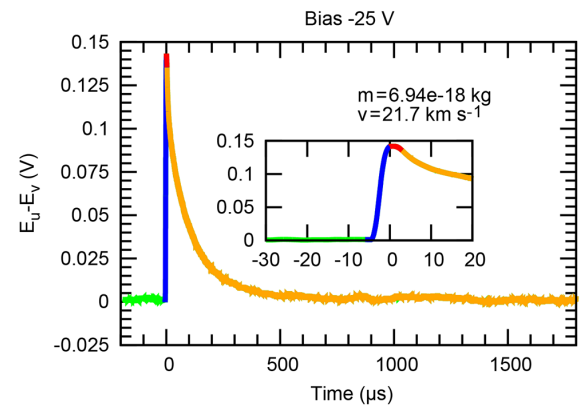

(b) U

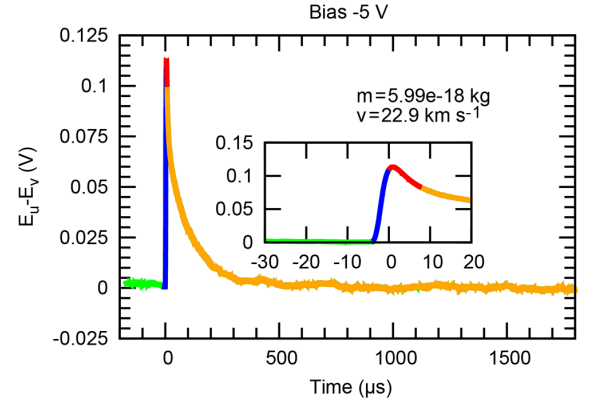

(c) $\cup_{0}$

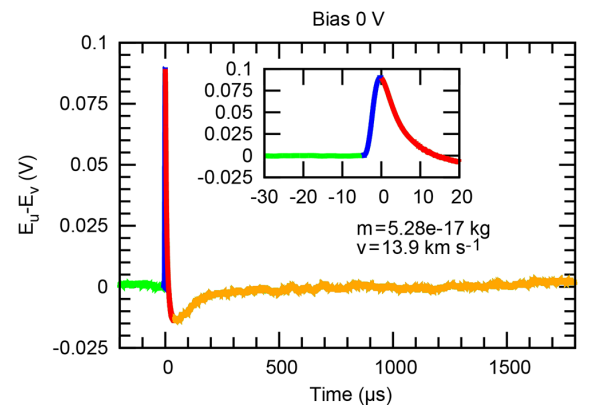

(d) $U_{+}$

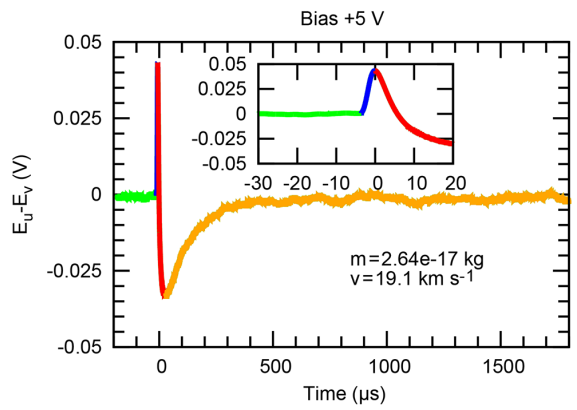

(e) $U_{+}$

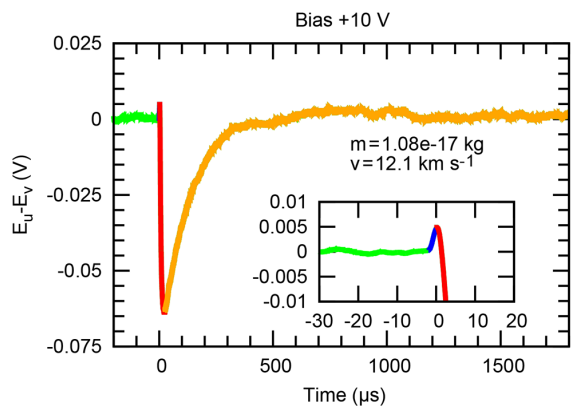

(f) $U_{++}$

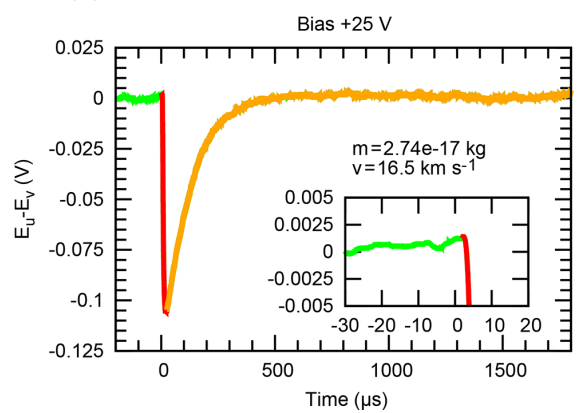

Figure 4. Laboratory simulation of dust impacts on the Cassini model showing the impact signal detected by the antenna ( $E_{U}$ boom was bombarded) for different polarities and sizes of bias voltage. Different phases of dust impact signal are distinguished by colours (green cloud generation, blue - electron escape, red - ion escape, orange - relaxation). The inserts show details of the pre-spikes (modified from Nouzák et al., 2018). The conditions in these laboratory measurements are comparable to a measurement in monopole configuration on the spacecraft.

Table 2. Examples of signal development for different biases (spacecraft potential) shown in Fig. 4.

\begin{tabular}{|c|c|c|c|c|c|}
\hline & (A) & (B) & (C) & (D) and (DD) & (E) \\
\hline $\begin{array}{l}\text { Process and current } \\
\text { (positive is toward } \\
\text { spacecraft) }\end{array}$ & $\begin{array}{l}U \text { negative } \\
U_{--}\end{array}$ & $\begin{array}{l}U \text { slightly negative } \\
U_{-}\end{array}$ & $\begin{array}{l}U \text { zero } \\
U_{0}\end{array}$ & $\begin{array}{l}U \text { slightly positive } \\
U_{+}\end{array}$ & $\begin{array}{l}U \text { positive } \\
U_{+}\end{array}$ \\
\hline $\begin{array}{l}\text { Electron recollection } \\
\text { Ion recollection } \\
\text { Electron escape } \\
\text { Ion escape } \\
\text { Relaxation }\end{array}$ & $\begin{array}{l}\text { zero } \\
\text { full } \\
\text { full - fast } \\
\text { close to zero } \\
\text { full }\end{array}$ & $\begin{array}{l}\text { suppressed } \\
\text { enhanced } \\
\text { enhanced } \\
\text { suppressed } \\
\text { reduced }\end{array}$ & $\begin{array}{l}\text { partial } \\
\text { partial } \\
\text { partial - fast } \\
\text { partial - slow } \\
\text { close to zero }\end{array}$ & $\begin{array}{l}\text { enhanced } \\
\text { suppressed } \\
\text { suppressed } \\
\text { enhanced } \\
\text { reduced }\end{array}$ & $\begin{array}{l}\text { full } \\
\text { zero } \\
\text { zero } \\
\text { full - slow } \\
\text { full }\end{array}$ \\
\hline
\end{tabular}


(red) is not apparent in this case. The electron part (blue) is followed directly by relaxation (orange).

- Panel (b) describes the signal shape for a reduced negative target potential. The number of escaping ions increases with the reduction of the negative potential (panel b). The electron part (blue) is followed by the smaller ion part (red) and relaxation (orange). A kink appears between the ion part (red) and relaxation (orange); see Fig. 3a.

- Panel (c), describing the signal measured at an unbiased target, shows that similar numbers of electrons and ions escape. The amplitude of the electron part (blue) is similar to the ion part (red).

- Panel (d) shows the case of positively charged target. The number of escaping electrons is reduced and the ion part of the signal exceeds the electron one. This results in a bipolar signal where the first part is typically called "pre-spike" (Collette et al., 2015; Thayer et al., 2016). A larger number of escaping ions change the polarity of the signal.

- Panel (e) shows the signal for a higher positive target bias potential - the first (electron) part of the bipolar pulse is reduced with increasing positive target potential.

- Panel (f) shows a case of even higher positive bias. All electrons are re-collected in this case. The signal has no electron (blue) part and it has no "pre-spikes" in this case.

The shapes of all pulses measured in the laboratory for various biases can be explained by the model described above. It must be noted, however, that since electron and ion escapes are very fast processes $(\sim \mu s)$, detection of a detailed structure of initial parts of pulses including "pre-spikes" thus requires fast electronics (sampling of the order of $100 \mathrm{kHz}$ ). Therefore, not all spacecraft are able to detect them, and a thorough inquiry into signal shapes using in situ data is difficult. The antenna signal can be also affected by the response of instrument electronics. For example, the transfer function of electronics can modify a shape and duration of the measured signal (Ye et al., 2019).

\section{Antenna signals observed in previous space missions}

Detection of dust impacts with antenna measurements has recently been done in several space missions. In the following, we discuss the major findings related to dust detection from the respective missions.

\subsection{STEREO}

STEREO is a NASA mission that was launched on 26 October 2006, with the study of coronal mass ejections as the primary scientific goal. The mission consists of two twin spacecraft that orbit the Sun at around $1 \mathrm{AU}$, one trailing the Earth (STEREO B) while the other leads (STEREO A). The study of the STEREO/WAVES radio receiver data proved to be of great interest for dust studies. STEREO/WAVES measured the flux of sub-micrometre dust near $1 \mathrm{AU}$ (Meyer-Vernet et al., 2009; Belheouane et al., 2012; Zaslavsky et al., 2012) and discovered a highly time-variable flux possible caused by nanometre-scale dust particles (Meyer-Vernet et al., 2009b). The nanodust impacts were observed frequently on both STEREO spacecraft as radio pulses on single monopole antennas. The physical mechanism that leads to their generation is not yet fully understood. The voltage was much higher on the antenna that was adequately located to be sensitive to impacts of prograde nanodust on each spacecraft (Meyer-Vernet et al., 2009b), which destabilised the photoelectron sheath of that antenna (Pantellini et al., 2012), producing a ratio between antenna voltages in agreement with the mechanism producing the pulses (Zaslavsky et al., 2012). The formation of the signal involves a transient local perturbation of the photoelectron equilibrium current on the antenna being close to the impact. The steps that lead to the antenna signals have been studied with plasma simulations and semi-empirically (see for example Pantellini et al., 2012; Meyer-Vernet et al., 2014; Zaslavsky, 2015). Kellogg et al. (2018) suggested that STEREO does not observe nanodust, but did not propose an alternative mechanism able to explain the observations. The larger dust impacts observed with STEREO/WAVES are observed with similar amplitudes at all three antennas. Based on STEREO/WAVES Zaslavsky (2015) proposed a model accounting for electric pulse generation by electron collection after an impact, linking the shape and amplitude of the electric signals to the dust and local plasma parameters. Figure 5 shows the model applied to typical impact clouds.

\subsection{Cluster}

The Cluster mission launched in 2000 consists of four identical spacecraft orbiting the Earth in close formation. The highly elliptical orbit (4-20 Earth radii) crosses various parts of the Earth's magnetosphere. Each spacecraft is equipped with two pairs of dipole electric field sensors (on $88 \mathrm{~m}$ booms tip-to-tip) (Gustafsson et al., 2001). The Wide Band Data (WBD) instrument provides data of a single electric or magnetic field component with a high sampling frequency in three modes $(27.4,54.9$ and $219.5 \mathrm{kHz}$ ) (Gurnett et al., 1997a). This resolution is sufficient to detect signals triggered by dust impacts. The dipole configuration is not sensitive to dust impacts on the spacecraft body. Some signal can be detected only after a direct dust impact on the one of the antennas or when the expanding impact cloud influences the 

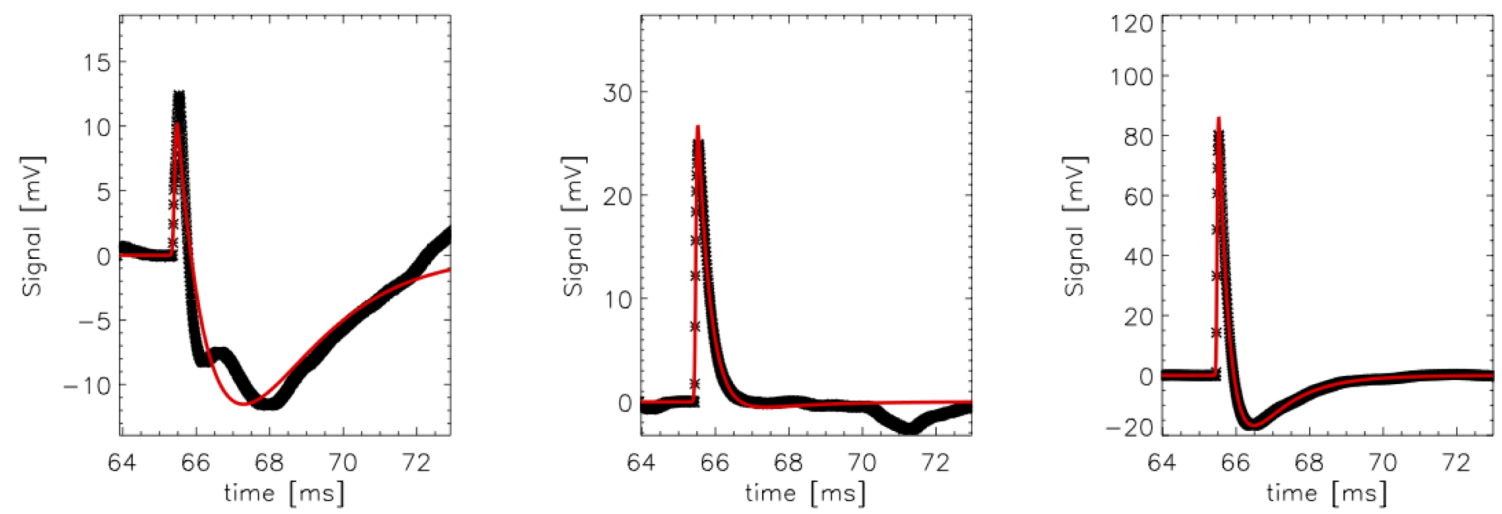

Figure 5. Dust impact signals recorded by the STEREO/WAVES TDS on STEREO A, shown with black crosses, are shown in comparison to a fit with a semi-empirical model, discussed in the text, shown with red solid lines (from Zaslavsky et al., 2015).

potential of the antenna. On the other hand, Cluster 1 operates with the only one remaining probe in the monopole configuration since 2009 (three probes have been lost during this time). This situation makes the detection of dust impacts by the Cluster 1 spacecraft possible (Vaverka et al., 2017a). On the other hand, a presence of a large number of natural waves including electrostatic solitary waves in the Earth's magnetosphere significantly complicates such detection (Vaverka et al., 2018). The fact that solitary waves are much more numerous than the expected amount of detected dust grains makes a reliable detection of dust impacts by the Cluster spacecraft very challenging. For this reason, the Cluster spacecraft are not optimal for dust studies.

\subsection{MMS}

The MMS mission consists of four Earth-orbiting spacecraft was launched in 2015 (Burch et al., 2016). While the missions are similar, the MMS electric field instruments differ just slightly from the Cluster ones. Each of the spacecraft is equipped with three pairs of electric field probes, two in the spin plane (120 m tip-to-tip) and one in the axial plane ( $29 \mathrm{~m}$; Torbert et al., 2016). The electric field is measured in the dipole configuration in all three directions with sampling frequency up to $8 \mathrm{kHz}$ (burst mode) and up to $256 \mathrm{kHz}$ in wave burst mode. The main difference is that the instrument operates simultaneously also in the monopole configuration. The combination of dipole and monopole measurements provides complex information about the ambient electric field and the spacecraft potential, which can be used for the reliable identification of dust impacts. Solitary waves and other structures in the ambient plasma or electric field generate pulses simultaneously in both monopole and dipole configuration. On the other hand, changes in the spacecraft potential triggered by the dust impact generate identical pulses on all monopole antennas and no signal in the dipole configuration (electric field data). This allows us to reliably distinguish changes in the spacecraft potential from the other pulses as solitary waves (see Vaverka et al., 2018). A measurement with MMS, shown in Fig. 6, illustrates the different detections in monopole and dipole configuration.

\subsection{Maven}

MAVEN is a NASA mission to Mars. It launched on 18 November 2013 and arrived at Mars on 22 September 2014. MAVEN is designed to study the escape of Mars's atmosphere, including the contribution of plasma processes associated with the interaction between the solar wind and the planet (Jakosky et al., 2015). Voltage spikes consistent with the impact of micron dust on the spacecraft were detected by the MAVEN LPW (Langmuir Probe and Waves) experiment at orbital altitudes between 200 and $1500 \mathrm{~km}$ (Andersson et al., 2015a). Andrews et al. (2015) found large variations in plasma density and spacecraft surface charging encountered by MAVEN as it dipped into the Martian ionosphere. This resulted in strong variation in the detectability of dust impact voltage spikes. Once these effects were taken into consideration, the estimated near-Mars micron dust flux observed by MAVEN was found to be consistent with the interplanetary dust flux expected at Mars (Andersson et al., 2015b). No evidence for moon-related dust rings or dust lifted from the surface (e.g. Sanchez-Lavega et al., 2015) was found with MAVEN LPW.

\subsection{Wind}

The NASA Wind spacecraft launched in November 1994 with the goal of studying the solar wind upstream of Earth. From 1994 to 2004, Wind executed a series of high apogee $\left(100 R_{\mathrm{e}}\right)$ orbits about Earth and several lunar flybys before being stationed in an orbit about the first Lagrange point $\left(L_{1}\right), \sim 250 R_{\mathrm{e}}$ sunward of Earth, where it remains operational to the present day (2019). The Wind WAVES experiment (Bougeret et al., 1995) detects voltage spikes consistent with the impact of micron-sized dust on the spacecraft body (Malaspina et al., 2014). These dust spikes are observ- 

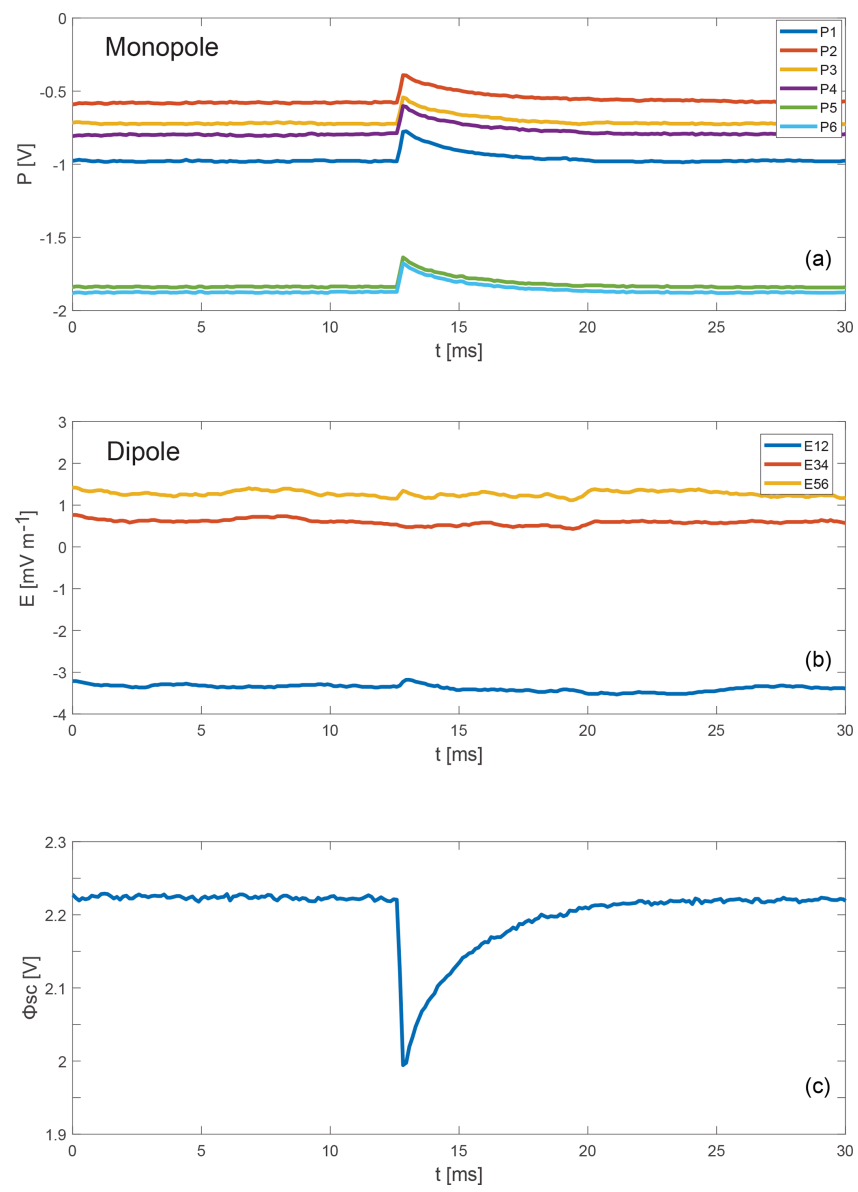

Figure 6. A potential signal of dust impact measured with MMS. Example of a typical event related to the change of the spacecraft potential observed in monopole configuration (a), the dipole measurements (b) with a lack of signal and the derived voltage pulse in (c) (see Vaverka et al., 2018).

able even though Wind WAVES makes only dipole electric field measurements, likely due to strong asymmetries of the dust impact signal on oppositely mounted antennas. Further, the rapid spin of the Wind spacecraft (one rotation every $3 \mathrm{~s}$ ) and asymmetry of dust impact voltage signals on the electric field wire antennas allows a crude directionality of the dust to be determined (Malaspina et al., 2014; Malaspina and Wilson, 2016). The observed amplitude and polarity of such signals are consistent with voltage induced on the antennas by positive ions produced by impacts on the spacecraft, after it has recollected the electrons (Meyer-Vernet et al., 2014); this new mechanism explained the previously unexplained voltage sign and amplitude for interstellar dust impacts on Wind, and also the absence of nanodust detection on this spacecraft. The yearly modulation of Wind-observed impacts was found to be consistent with the yearly variation in interplanetary micron dust (Malaspina et al., 2014; Wood et al., 2015). Further supporting this conclusion was the observation that both Wind and STEREO observe the same yearly modulation of interstellar dust flux (Kellogg et al., 2016). The long duration of the Wind mission (>25 years, over two full solar cycles) presents a unique opportunity to study how the solar magnetic field modulates the entry of interstellar dust into the solar system and its arrival at $1 \mathrm{AU}$. To facilitate such studies, a database cataloguing all dust impacts observed by Wind was created (Malaspina and Wilson, 2016) and made publicly available through the NASA Space Physics Data Facility Coordinated Data Analysis Web (CDAWeb) (https://cdaweb.sci. gsfc.nasa.gov/index.html/, last access: 28 November 2019).

\subsection{Cassini}

The Cassini Radio and Plasma Wave Science (RPWS) instrument measures oscillating electric fields over the frequency range $1 \mathrm{~Hz}$ to $16 \mathrm{MHz}$ and magnetic fields in the range $1 \mathrm{~Hz}$ to $12 \mathrm{kHz}$ (Gurnett et al., 2004). The instrument uses three nearly orthogonal electric field antennas $\left(E_{u}, E_{v}, E_{w}\right.$, each $10 \mathrm{~m}$ long and $2.86 \mathrm{~cm}$ in diameter) and three orthogonal magnetic search coil antennas. The $E_{u}$ and $E_{v}$ antennas are often used together as a dipole antenna and $E_{w}$ and the spacecraft body as a monopole antenna (Gurnett, 1998), both sensitive to dust impacts. The south-polar plume of Enceladus was one of the top discoveries made by the Cassini mission. During the Enceladus plume crossing, besides dust impact signals, RPWS detected plasma oscillations induced by dust impacts, the frequencies of which are equal to the local plasma frequencies (Ye et al., 2014), which can be explained by a beam-plasma instability induced by the impactproduced electrons when their speed exceeds the thermal speed of the ambient plasma (Meyer-Vernet et al., 2017). Comparison of observations (Ye et al., 2014b) showed that the dust density profile measured by RPWS is consistent with that measured by the dedicated dust detector on board.

Cassini allowed for a comparison of measurements in dipole and monopole configuration. The difference is clearly seen in Fig. 7, which shows the electric power spectrum measured by the Cassini RPWS HFR receiver simultaneously in dipole (top) and monopole (bottom) mode in Saturn's E-ring at the first close approach of Enceladus (Meyer-Vernet et al., 2014). During the subsequent mission, the Wideband Receiver (WBR) of the RPWS instrument was switched from monopole mode to dipole mode at a ring plane crossing, so that the responses of these two antenna modes to dust impacts were compared, assuming the dust density and size distribution did not change across the ring plane (Ye et al., 2016a). Figure 8 shows an RPWS wave power spectrogram, which covers a $1 \mathrm{~h}$ period around a ring plane crossing on DOY 001, 2016. As the antenna mode switched from monopole to dipole at the ring plane at $\sim 10: 30$ UTC, the spectral power decrease was accompanied by a significant decrease in the negative impact rates (blue) and the polarity ratio jumping to $\sim 1$. The spectral power is proportional to the product of impact rate and average voltage jump size squared (MeyerVernet, 1985). So, the difference in spectral power at the an- 


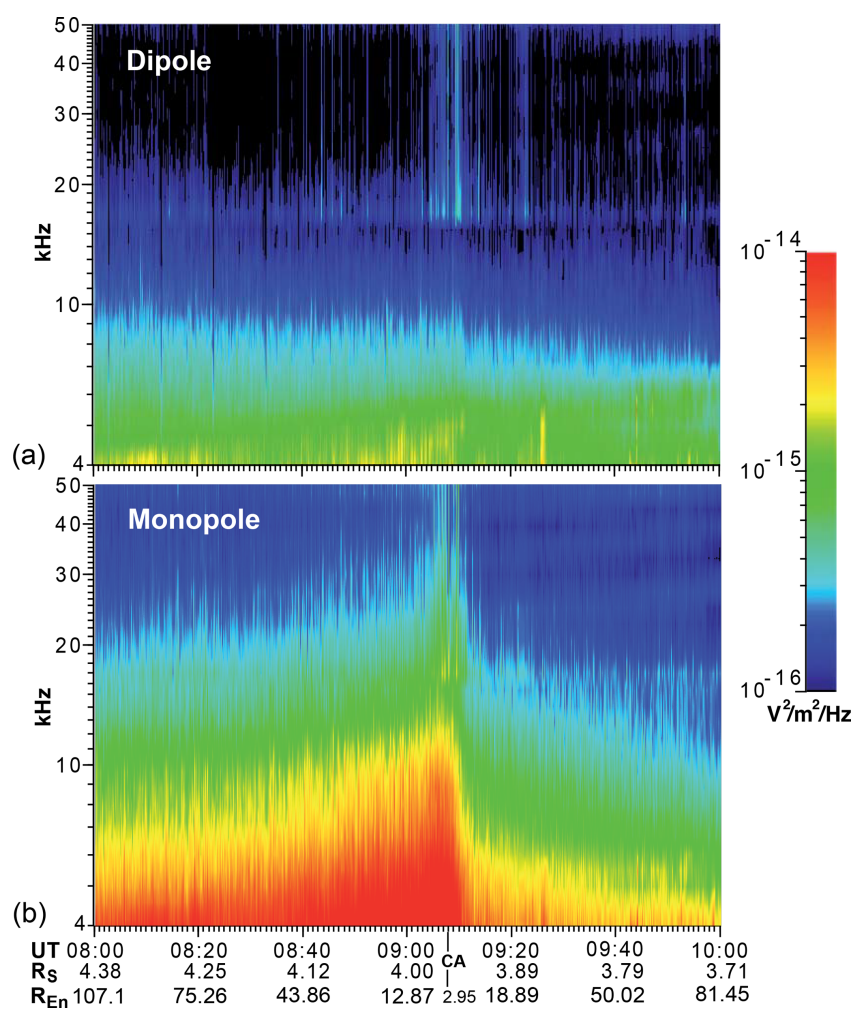

Figure 7. From Meyer-Vernet et al. (2014): time-frequency electric power spectral density measured by Cassini/RPWS on 9 March 2005 in Saturn's E ring, in dipole (a) and monopole mode (b). The increase due to micron-sized dust impacts on the spacecraft only appears in monopole mode, whereas the dipole only measures the weaker plasma quasi-thermal and impact noise.

tenna switch could be due to either a lower impact rate or smaller average voltage pulse size, or both.

In Fig. 9, we show a comparison of the vertical dust density profiles measured by the RPWS WBR and the Cassini Cosmic Dust Analyzer (CDA) High Rate Detector (HRD) during the ring plane crossing on DOY 361, 2016. HRD uses polarised foils for dust detection and can measure high impact rates of particles bigger than a size threshold that depends on the impact speed (Srama et al., 2004). Discontinuities in the RPWS dust density profile are due to gain changes of WBR. The CDA data showed consistent peak densities of around $0.04 \mathrm{~m}^{-3}$ (threshold $\sim 0.8 \mu \mathrm{m}$ ) during the ringgrazing orbits, less than 1 order of magnitude higher than the RPWS dust density, which is within the uncertainty limit of the method (Ye et al., 2014). The density peak measured by RPWS is wider than that by CDA with a full-width halfmaximum of $600-1000 \mathrm{~km}$ compared to $475 \mathrm{~km}$ for the latter (averaged profile shows a FWHM of $475 \mathrm{~km}$ ). This difference is discussed in detail in Ye et al. (2018a). The E ring density structure based on RPWS measurements has been shown to be consistent as well with that revealed by optical observation (Ye et al., 2016a).
Ye et al. (2016b) compared the data collected with these two antenna setups and found that the wave power spectral density observed by the monopole antenna is $\approx 10 \mathrm{~dB}$ higher than that observed by the dipole antenna. This does not necessarily mean that the monopole antenna is more sensitive to individual dust impacts, because direct comparison of the waveforms observed by these two antennas showed that the sizes of the voltage jumps induced by dust impacts are comparable. Comparison of the impact rates showed that the monopole antenna detects $\sim 10$ times more dust impacts than the dipole antenna. This difference in impact rates is roughly in line with the difference in the effective impact areas of the spacecraft body and the dipole electric antenna. Detailed analysis showed that the polarity ratio of the impacts detected by the dipole antenna changes with the projected area ratio of the dipole antenna elements $\left(E_{u}\right.$ and $\left.E_{v}\right)$ as the spacecraft rotates, providing strong evidence that the dipole mode primarily detects impacts on the antenna booms.

Cassini cruise measurements between 1 and $5 \mathrm{AU}$ also enabled us to study the rise time of the impact ionisation pulses as a function of dust mass and of heliocentric distance (Meyer-Vernet et al., 2017), a quantity of great importance for future missions since it determines the frequency range and voltage amplitude for dust detection.

\section{Dust in the inner heliosphere}

Many dust observations describe the dust flux near-Earth orbit; it can be estimated from meteor observations, crater statistics and measurements from spacecraft. Based on these sources, an empirical polynomial mass distribution was found (Grün et al., 1985; Ceplecha et al., 1998). Observations by the STEREO spacecraft allowed this distribution to be extended to smaller masses (Zaslavsky et al., 2012; Meyer-Vernet et al., 2009b; Malaspina et al., 2015). There is still an uncertainty, however, about the absolute flux values. Estimates of cosmic dust fluxes near $1 \mathrm{AU}$ and onto Earth range over several orders of magnitude and are based on a number of different assumptions (cf. for example Nesvorný et al., 2011; Mann et al., 2011; Plane, 2012). There is even less known on the dust flux inside $1 \mathrm{AU}$ and estimates are often based on extrapolation of the flux curve obtained near Earth and considering the major forces acting on dust particles (Mann et al., 2004): Large dust particles ("micron dust" $m>10^{-14} \mathrm{~kg}$ ) are mainly influenced by gravity force and move in Keplerian orbits superimposed by a slow migration inward caused by the Poynting-Robertson effect. For dust with masses $10^{-19} \mathrm{~kg}<m<10^{-14} \mathrm{~kg}$ ("beta meteoroids") the radiation pressure force is comparable to the gravitation; when these small particles form, typically by collisions of larger dust, they move outward in hyperbolic orbits (Czechowski and Mann, 2007). For even smaller dust with $m<10^{-19} \mathrm{~kg}$ (nanodust) electromagnetic forces prevail, and they are deflected in a way that is similar to the pick-up pro- 

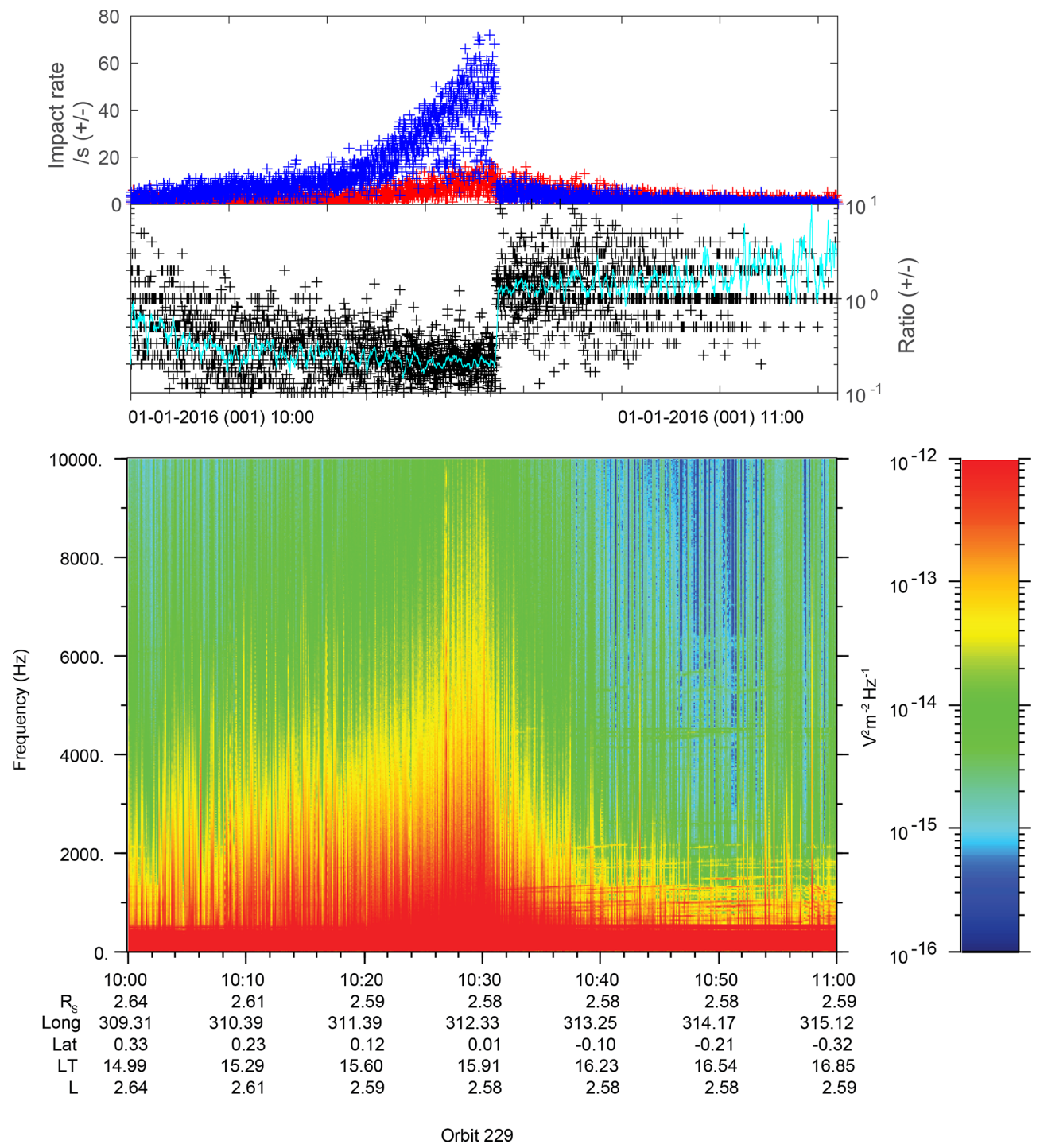

Figure 8. Adapted from Figs. 4 and 5 of Ye et al. (2016b). RPWS wave power spectrogram around a ring plane crossing on DOY $001,2016$. The top panel shows the positive (red) and negative (blue) impact rates. The middle panel shows the impact signal polarity ratios with the moving averages (teal). At $\sim$ 10:30 UTC, the antenna used was switched from monopole to dipole, which was accompanied by a decrease in the spectral power and the polarity ratio jumping back to 1 .

cess of ions that newly form in the solar wind (Mann et al., 2010).

The observational studies of scattered light and thermal emission from the dust are constrained by large contributions from dust near the Earth to the brightness because the observed brightness is an integrated signal along the line of sight (Mann et al., 2004). Since early infrared eclipse observations (MacQueen, 1968) showed irregularities in the slope of the corona brightness with varying distance from the solar limb, the possibility of dust rings existing around the Sun is discussed. Indeed, model calculations were made to show that dust rings can form for specific dust properties in the initial stage of dust sublimation when the dust size is reduced and the radiation pressure force increases with reduced dust size (Mukai and Yamamoto, 1979). The solar eclipse observations made over the years suggest, however, that the observed features can be explained without the existence of pronounced rings (Mann, 1992) and that the average dust properties in the inner heliosphere change over timescales of years (Kimura et al., 1997; Ohgaito et al., 2002). The spatial distribution of the dust that can be derived from the scattered light and thermal emission observations suggests that the dust number density increases with distance from the Sun, and in combination with the increasing orbital velocities this leads to increasing dust flux inversely proportional to the distance $r$ from the Sun within or close to the ecliptic plane (Mann et al., 2004). The amount of dust above the solar poles and in orbits with high inclination is even less 


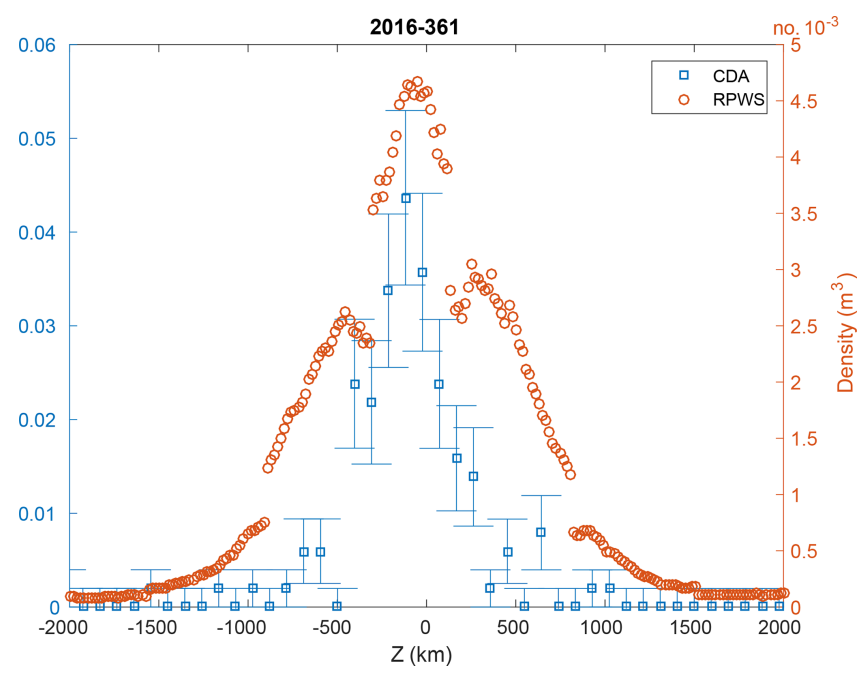

Figure 9. Adapted from Fig. 4 of Ye et al. (2018a). Comparison of vertical dust density profiles of the Janus-Epimetheus ring measured by RPWS and CDA during the ring plane crossing on DOY 361, 2016. There is 1 order of magnitude of difference between the two results, which is within the uncertainty limit estimated for the RPWS measurement (Ye et al., 2014).

known (Mann et al., 2004). Recent white light observations from STEREO A (Stenborg and Howard 2017a, b) provide the shape of the F corona and inner zodiacal light from 5 to $24^{\circ}$ line-of-sight elongation and show its flattening to larger elongation. Closer analysis also showed that the flattening varied with spacecraft position, indicating an influence of the dust brightness near the spacecraft (Stenborg, et al., 2018; Stauffer et al., 2018), again showing the importance of considering the line-of-sight effects when analysing brightness observations.

While we can expect that the flux of large particles in the inner solar system increases proportionally to $1 / r$, where $r$ ist the distance from the Sun, it is difficult to predict the flux of smaller dust. Detailed trajectory calculations show that nanodust can be trapped in orbits with perihelia very close to the Sun instead of being ejected (Czechowski and Mann, 2010). Trapping conditions depend on a number of different parameters so that the nanodust flux outward can vary in time (Czechowski and Mann, 2010, 2012). The majority of nanoparticles and those that form at distances $0.2 \mathrm{AU}$ from the Sun or larger are usually ejected outward. Figure 10 shows the velocity of nanoparticles that are ejected from circular orbit at $0.2 \mathrm{AU}$. While the particles gradually gain speed, one can see that in the inner solar system they still have a velocity close to that of the parent object. The trajectory of the largest particle shown in the figure with approximate radius $100 \mathrm{~nm}$ corresponds to a beta-meteoroid that is mainly influenced by radiation pressure force (Wehry and Mann, 1999) because it has a smaller value of surface charge to mass than the smaller particles. Figure 11 shows the velocity as a function of distance for the same range of

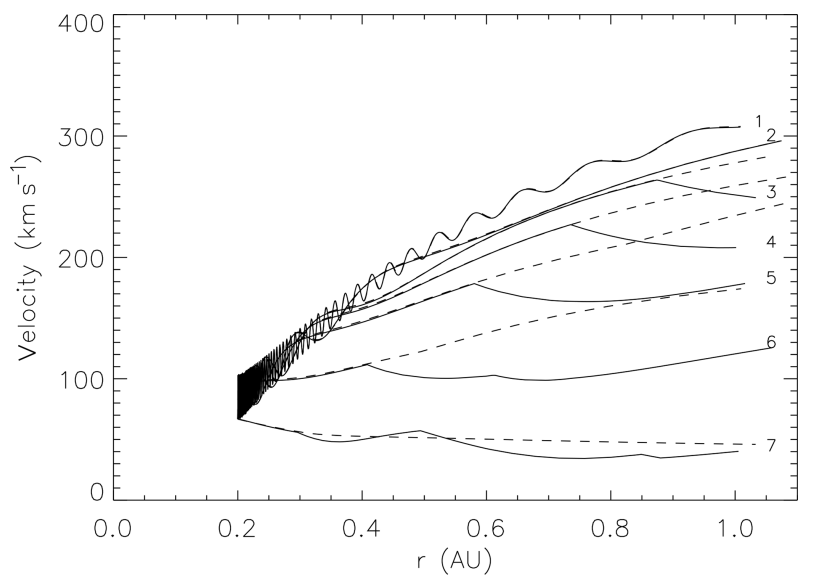

Figure 10. Velocity as function of distance from the Sun for particles with $Q / m=10^{-4}, 10^{-5}, 10^{-6}$ and $10^{-7} \mathrm{e} / \mathrm{m}_{\mathrm{p}}$ released from a circular orbit with the radius $0.2 \mathrm{AU}$ near the ecliptic plane. Solid lines correspond to the focusing, and dashed to defocusing, magnetic field orientation (adapted from Mann et al., 2014).

parameters for the nanodust but when particles are released from initially highly elongated orbits. The orbital eccentricity and perihelion assumed for the model calculations presented correspond to the orbits of the Aquarid meteoroids and the nanoparticles are released at different locations of the orbit. One can see that their trajectories in the inner solar system strongly depend on the initial condition.

In addition to uncertainty of the dust trajectories, the dust production rate is hard to predict. The majority of dust particles smaller than micron size inside $1 \mathrm{AU}$ are produced by fragmentation during collisions of larger dust particles. The dust formation by mutual collisions depends on the dust material compositions (Ishimoto and Mann, 1999; Mann and Czechowski, 2005); it varies with the dust velocities and it is for instance enhanced when coronal mass ejections push out nanoparticles (Czechowski and Kleimann, 2017). Sun grazing comets (cf. Jones et al., 2018) are another source of time variable dust flux. The nanodust flux can also vary due to other effects, like the variation of the source, e.g. when the dust flux is enhanced by a single collision event in the inner heliosphere, or due to the influence of the solar magnetic field structure (see Czechowski and Mann, 2012; Juhasz and Horanyi, 2013). While estimates are made for time-stationary conditions, current sheet crossings occur along the trajectories and, in addition, the magnetic field is time-variable. Coronal mass ejections also change the conditions, pushing large fractions of nanodust outward at speeds reaching $1000 \mathrm{~km} \mathrm{~s}^{-1}$ (Czechowski and Kleimann, 2017).

From 10 years of STEREO A observations attributed to nanodust impacts several important properties can be obtained. The signal explained as nanodust is 10-100 times more frequent in a stream interaction region (SIR) or during interplanetary coronal mass ejections (ICMEs). The observed signals exhibited a periodicity due to the crossing by 

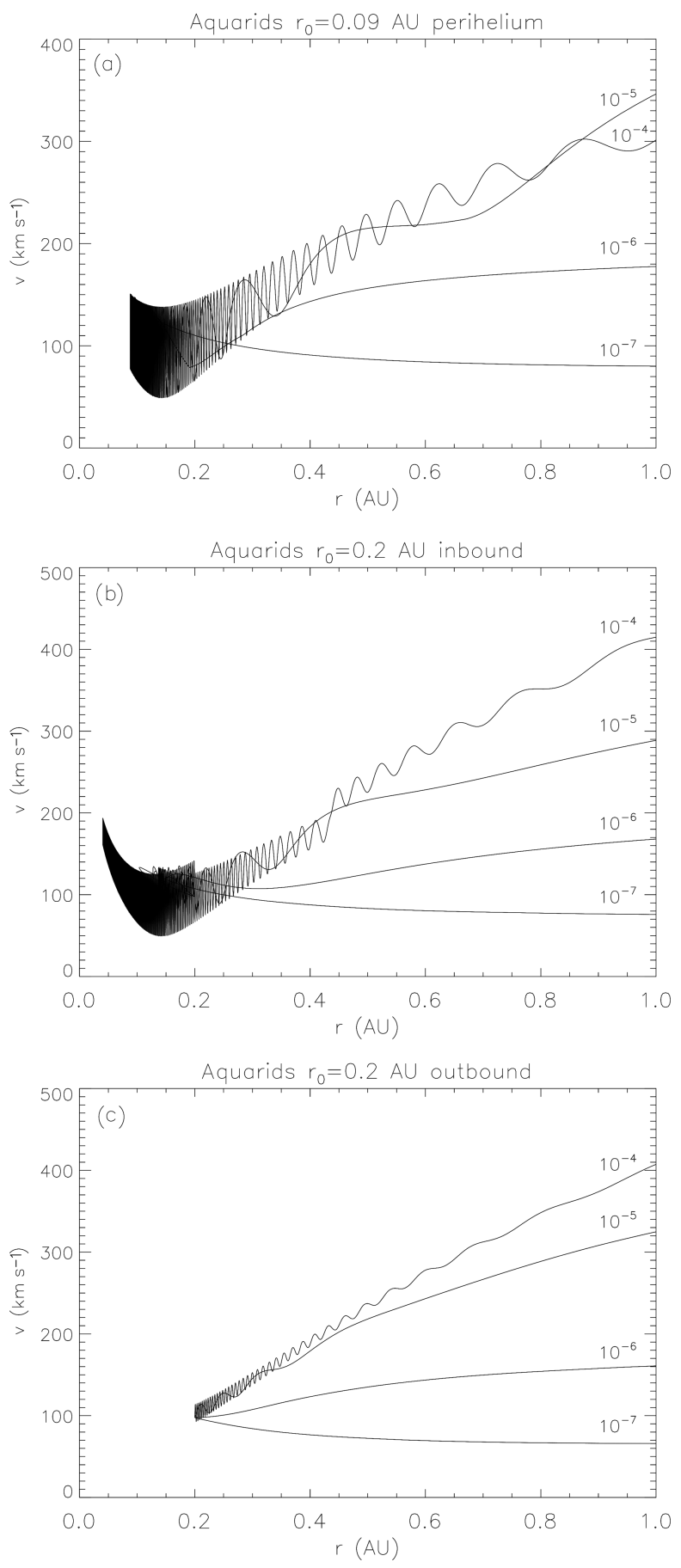

Figure 11. Velocities of small fragments released from Aquarid meteoroids as a function of the heliocentric distance. The velocities are calculated for dust with $Q / m=10^{-4}, 10^{-5}, 10^{-6}$ and $10^{-7} e / m_{\mathrm{p}}$. The ratio of surface charge to mass corresponds to sizes $3 \mathrm{~nm}, 10 \mathrm{~nm}$ and larger. The particles are released from the orbit of Aquarids at the perihelion $(0.09 \mathrm{AU}$ from the Sun, a), and at the distance $0.2 \mathrm{AU}$ on the inbound (b) and outbound (c) parts of the orbit. From Czechowski and Mann (2018).

STEREO of the solar magnetic equator. A correlation with solar wind perturbations and periodicities corresponding to those of Mercury and Venus were also detected (Le Chat et al., 2015). These signals nearly disappeared on STEREO A around 2012 (Le Chat et al., 2013; Malaspina et al., 2015) when the heliosphere entered a defocusing configuration in which the nanodust coming from the inner heliosphere are pushed away from the solar magnetic equator, therefore possibly preventing their observation. Observations with the RPWS instrument on board Cassini between 1 and $5 \mathrm{AU}$ have produced two further important properties of interplanetary nanodust. Firstly, the average nanodust flux measured at $1 \mathrm{AU}$ was similar in order of magnitude to the average of the highly variable flux measured by STEREO when the heliosphere was in a focusing configuration (Schippers et al., 2014), and it decreased roughly as the inverse squared heliocentric distance (Schippers et al., 2015). Secondly, the nanodust fluxes were found to follow the variation in solar wind drift speed closely (Meyer-Vernet et al., 2017) as predicted by nanodust dynamics (Mann and Czechowski, 2012).

Finally, aside from providing information on dust in the inner heliosphere, it is also quite possible that the Parker Probe and Solar Orbiter missions will find more effects that dust particles have on the solar wind. Cosmic dust particles interact with the surrounding plasma through electric charge collection, the photoelectric effect (Mann et al., 2014) and destruction processes (sputtering, fragmentation, sublimation). Photoionisation, electron-impact ionisation and charge exchange quickly ionise the atoms and molecules in the solar wind (Mann and Czechowski, 2005) so that dust destruction generates pick-up ions. While those interactions do not affect solar wind measurable parameters much near $1 \mathrm{AU}$ (Mann et al., 2010), conditions are possibly different near the Sun. Dust particles sublimate at bulk temperatures $\sim 1000-2000 \mathrm{~K}$ inside $\sim 10$ solar radii (Mukai and Mukai, 1973; Mann et al., 2004; Mann and Murad, 2005). A fraction of dust material vaporises during collision (Mann and Czechowski, 2005). The effect of dust on the solar wind is also time-variable, as for instance the dust destruction rates due to sputtering increase during coronal mass ejections (Ragot and Kahler, 2003). The solar wind particles also change charge state by interaction with the dust surface or by passing through the particles (Mann et al., 2010; Minato et al., 2004). And some authors suggest that newly formed charged dust fragments generate features in the solar wind magnetic field (Connors et al., 2014; Lai et al., 2013, 2015).

\section{Discussion of implications for observations with Parker Probe and Solar Orbiter}

The design of the radio and plasma waves instrument (RPW) on the ESA Solar Orbiter (Mueller and al., 2019) is similar to STEREO/WAVES instrumentation. The electric antenna system on RPW consists of a set of three identical anten- 


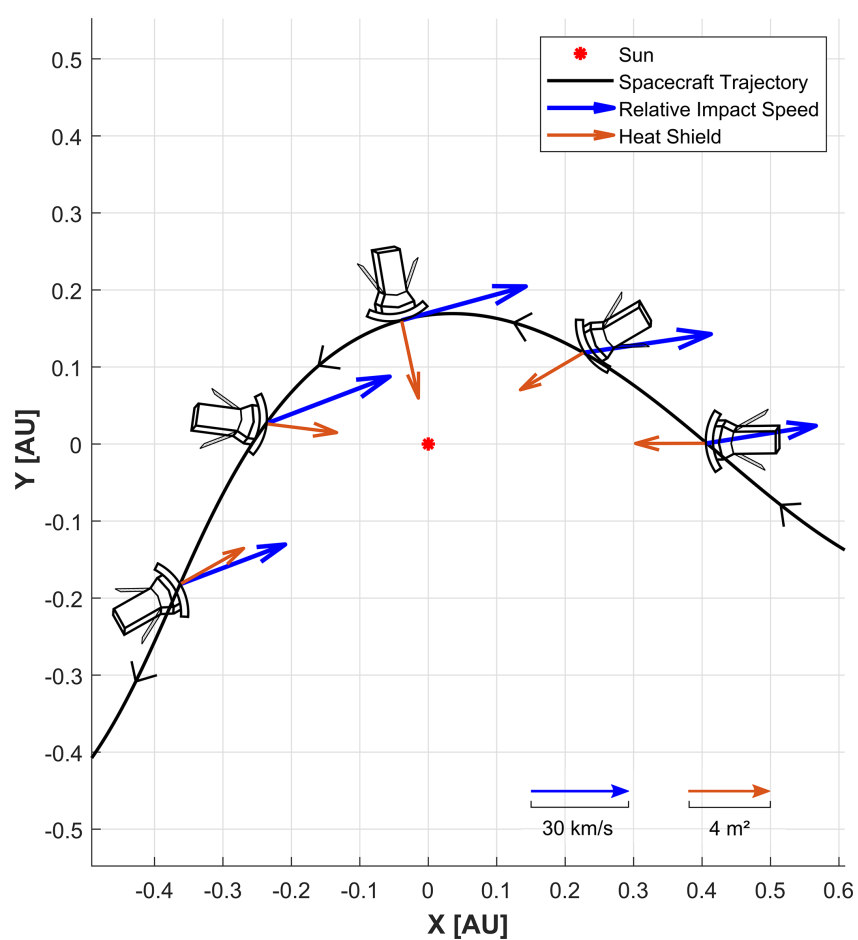

Figure 12. Parker Solar Probe trajectory during first perihelion passage. Blue arrows indicate speed of spacecraft relative to the dust particles in circular orbit. Red arrows indicate the surface vector of the spacecraft heat shield. Figure courtesy of Calvinhac (2019).

nas deployed from $+Z$ axis and from the opposite corners of the spacecraft and can operate in dipole and monopole modes. RPW antennas consist each of a $1 \mathrm{~m}$ rigid deployable boom and a $6.5 \mathrm{~m}$ stacer deployable monopole, which has a $1.5 \mathrm{~cm}$ radius. The Time Domain Sampler (TDS) subsystem of the RPW instrument (Maksimovic et al., 2019) is designed to capture electromagnetic waveform snapshots at high cadence from $200 \mathrm{~Hz}$ to $200 \mathrm{kHz}$, resolving in particular voltage spikes associated with interplanetary dust impacts.

Solar Orbiter will make observations of the Sun and in situ measurements from elliptic orbits coming as close as $\sim 60$ solar radii $(\sim 0.285 \mathrm{AU})$ to the Sun. The aphelia lie outside $0.8 \mathrm{AU}$ for a large part of the 7-year nominal mission time during which orbital latitude reaches $25^{\circ}$. The long cruise phase of Solar Orbiter and the elongated spacecraft orbits with aphelia close to $1 \mathrm{AU}$ provide the opportunity to study in detail the dust flux near $1 \mathrm{AU}$ and to estimate the flux of submicrometre dust onto Earth, its time variation and its variation during part of a solar cycle.

The FIELDS instrument on Parker Solar Probe (Bale et al., 2016) combines magnetic and electric field measurements into a single, coordinated experiment. Four electric field antennas ( $2 \mathrm{~m}$ long, $3.18 \mathrm{~mm}$ diameter Niobium C-103 thinwalled tubes) are mounted at the base of the heat shield, and deploy in full sunlight out of the spacecraft wake, while a fifth antenna is mounted on the magnetometer boom in
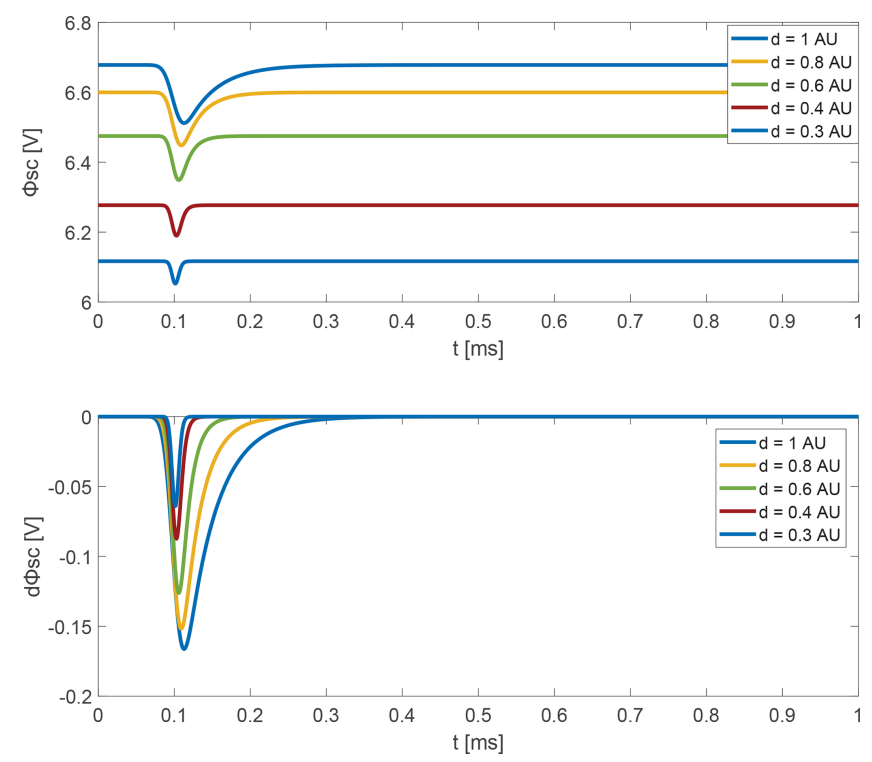

Figure 13. The estimated signals for impacts of $0.1 \mu \mathrm{m}$ particles with speed $100 \mathrm{~km} \mathrm{~s}^{-1}$ for spacecraft at different distance from the Sun. The charge production of the impact is assumed to be $Q=$ 30 pC. Estimate based on the Vaverka et al. (2017b) model.

the umbra of the spacecraft. The sensor electric field signals are transferred to a Digital Fields Board (DFB), a Time Domain Sampler (TDS) and a Radio Frequency Spectrometer (RFS) for signal processing and digitisation. The DFB and TDS make rapid samples of waveforms with the highest sampling rate of 150000 samples per second (DFB) and 2000000 samples per second (TDS), with an on-board selection of events to reduce bit rate. The low frequency (LF) part of the RFS is a dual channel digital spectrometer receiving inputs from the four first antennas, either in dipole or monopole mode, with a frequency range of $10 \mathrm{kHz}$ to $2.4 \mathrm{MHz}$, allowing a relative frequency spacing of about $4.5 \%$.

Parker Solar Probe orbits the Sun in the ecliptic plane, making seven Venus gravity assist manoeuvres during the 7year nominal mission duration, which will lessen its perihelia to less than $10 R_{\mathrm{S}}$, the closest any spacecraft has come to the Sun. In this way, the spacecraft will spend a total of $937 \mathrm{~h}$ inside $20 R_{\mathrm{S}}, 440 \mathrm{~h}$ inside $15 R_{\mathrm{S}}$ and $14 \mathrm{~h}$ inside $10 R_{\mathrm{S}}$ (Fox et al., 2015). The surrounding plasma changes considerably along the spacecraft orbits (Bale et al., 2016). The orbital trajectory for the first orbit around the Sun is shown in Fig. 12.

Our considerations suggest that both RPW and FIELDS measurements in monopole mode will be able to detect signals generated by dust impacts. Distinction between dust and other wave features needs to be considered based on the observational data. At present, we do not know the mass range of dust particles that will be detectable. As heliocentric distance decreases, the pulse's decay time will decrease faster than its rise time (Meyer-Vernet et al., 2017), eventu- 

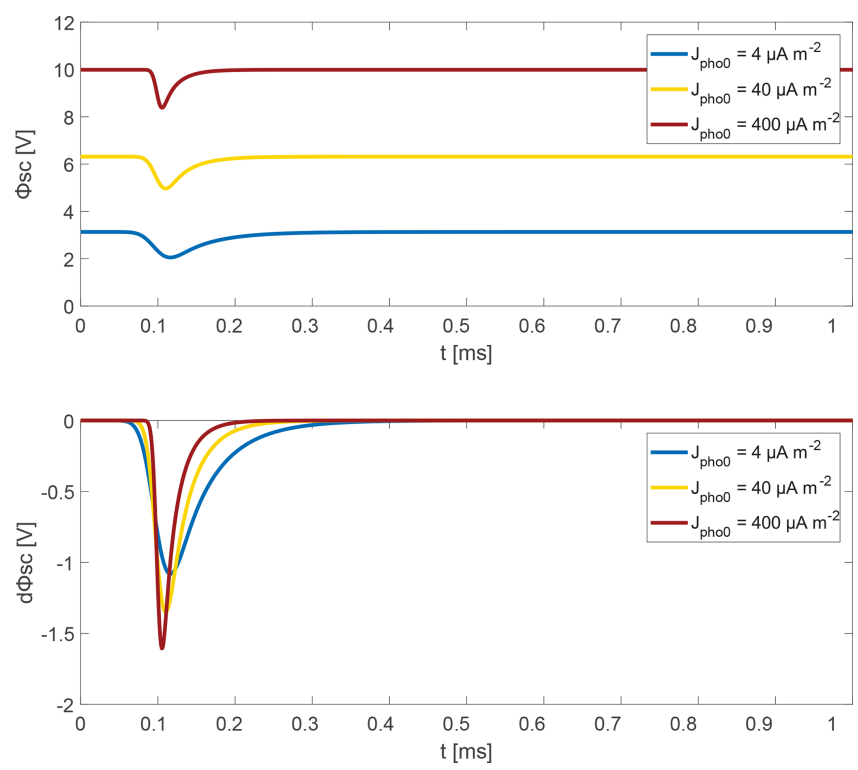

Figure 14. The spacecraft potential change during dust impact for different values of the photocurrent. A typical value of photocurrent at $1 \mathrm{AU}$ is $40 \mu \mathrm{A} \mathrm{m}^{-2}$. While its influence on spacecraft potential is considerable, this is not for the change induced by impacts.

ally becoming smaller than the rise time, which will decrease the dust signal for large grains. The spacecraft charging and charged particle dynamics close to the Sun are expected to be considerably complicated by the presence of a potential barrier (sheath structure) due to strong photoemission (Ergun et al., 2010; Campanell, 2013) as well as by the presence of the thermal shield and non-conducting solar panels.

Vaverka et al. (2017b) simulated pulses generated by dust impacts in various plasma environments using a simple numerical model. The spacecraft potential is calculated using orbital-motion-limited theory and the current generated by the dust impact is represented by a Gaussian function. We used this approach to simulate the spacecraft charging in the inner solar system. The rise time of the pulse was estimated according to Meyer-Vernet et al. (2017) for variable photoelectron sheaths. The rise time of the measured pulse is also affected by the response of the antenna electronics. These electronic effects were not taken into account in the model. This model also does not describe the detailed structure of the pulses including "pre-spikes" but only their general shapes, and it can be appropriate to estimate the conditions for dust impacts. Figure 13 shows estimated signals for impacts of $0.1 \mu \mathrm{m}$ particles with speeds of $100 \mathrm{~km} \mathrm{~s}^{-1}$ for spacecraft at different distances from the Sun. The top panel represents temporal evolution of the spacecraft potential and the bottom panel shows changes in the equilibrium potential. The charge production of the impact is assumed to be $Q=30 \mathrm{pC}$ according the equation presented in Sect. 2. The amplitude of the pulse is then proportional to the mass of the impinging grain and to its velocity with power between 2.5 and 6.2 (de- pends on materials). It is possible to see that the amplitude and duration of the pulses are reduced with decreasing distance from the sun. This fact means that the sensitivity of dust impact detection is smaller close to the sun. It is necessary to mention that the conditions for the orbital-motion-limited theory are not satisfied near the sun. The presence of the potential barrier created due to strong photoemission, described by Ergun et al. (2010) and Campanell (2013), strongly influences the spacecraft charging and charge dynamics. An interesting result is that the shape of the detected signal depends only weakly on the solar UV illumination, which leads to the photocurrent as shown in Fig. 14 for $1 \mathrm{AU}$ from the sun. The increase or decrease in the solar activity, which varies the UV flux, influence the spacecraft potential but not so much the profile of the pulse generated by dust impacts.

A challenge in the data analysis will be to distinguish dust impact signals from other events. A comparison to dust measurements from other spacecraft at a similar distance from the Sun as Parker Probe and Solar Orbiter should be considered. BepiColombo equipped with the Mercury Dust Monitor (MDM, Nogami et al., 2010) and electric field antennas (PWI, plasma wave investigation; Kasaba et al., 2010) will in the near future study the dust environment near Mercury at 0.31 to $0.47 \mathrm{AU}$ from the Sun. Though noise events are also considered an issue for those measurements, they offer an opportunity for simultaneous dust observations from several spacecraft so as to have a more reliable distinction of dust impacts and other events.

Data availability. The data will be published for open access on the UiT Open Research Repository at https://doi.org/10.18710/ LURI1R (Mann et al., 2019).

Author contributions. This paper was prepared during discussions of the ISSI team on dust impacts at the International space science institute in Bern, Switzerland. All authors contributed to the writing of this text in an open editing process and to the discussions that lead to the writing.

Competing interests. The authors declare that they have no conflict of interest.

Acknowledgements. This work is developed at the International Space Science Institute, ISSI, in Bern in a team on dust impacts in spacecraft. We thank Louis Calvinhac from University Toulouse III for preparing Fig. 12 during a student project carried out at UiT.

Financial support. This research has been supported by the Research Council of Norway (grant no. 262941), the Czech ministry of education youth and sport (grant no. LTAUSA 17066), NASA (grant no. 1415150) and CDAP (grant no. 14397000). 
Review statement. This paper was edited by Dominique BockeleeMorvan and reviewed by Laila Andersson and one anonymous referee.

\section{References}

Andersson, L., Ergun, R. E., Delory, G. T., Eriksson, A., Westfall, J., Reed, H., and Meyers, D.: The Langmuir probe and waves (LPW) instrument for MAVEN, Space Sci. Rev., 195, 173-198, 2015a.

Andersson, L., Weber, T. D., Malaspina, D., Crary, F., Ergun, R. E., Delory, G. T., Andrews, D. J., Horanyi, M., Collette, A., Yelle, R., and Jakosky, B. M.: Dust observations at orbital altitudes surrounding Mars, Science, 350, aad0398, https://doi.org/10.1126/science.aad0398, 2015b.

Andrews, D. J., Andersson, L., Delory, G. T., Ergun, R. E., Eriksson, A. I., Fowler, C. M., McEnulty, T., Morooka, M. W., Weber, T., and Jakosky, B. M.: Ionospheric plasma density variations observed at Mars by MAVEN/LPW, Geophys. Res. Lett., 42, 88628869, 2015.

Auer, S.: Instrumentation, in: Interplanetary Dust, edited by: Grün, E., Springer, New York, 385-444, 2001

Bale, S. D., Goetz, K., Harvey, P. R., Turin, P,Bonnell, J. W., Dudok de Wit, T., Ergun ,R. E., MacDowall, R. J., Pulupa, M., Andre, M., Bolton, M., Bougeret, J.-L.,Bowen, T. A., Burgess, D., Cattell, C. A., Chandran, B. D. G., Chaston, C. C., Chen, C. H. K., Choi, M. K., Connerney, J. E., Cranmer, S., Diaz-Aguado, M., Donakowski, W., Drake, J. F., Farrell, W. M., Fergeau, P., Fermin, J., Fischer, J., Fox, N., Glaser, D., Goldstein, M., Gordon, D., Hanson, E., Harris, S. E., Hayes, L. M., Hinze, J. J., Hollweg, J. V., Horbury, T. S., Howard, R. A., Hoxie, V., Jannet, G., Karlsson, M., Kasper, J. C., Kellogg, P. J., Kien, M., Klimchuk, J. A., Krasnoselskikh, V. V., Krucker, S., Lynch, J. J., Maksimovic, M. ,Malaspina, D. M. ,Marker, S., Martin, P., Martinez-Oliveros, J., McCauley, J., McComas, D. J. T., McDonald, N., Meyer-Vernet, M., Moncuquet, S. J., Monson, F. S., Mozer, S. D., Murphy, J., Odom, R., Oliverson, J., Olson, E. N., Parker, D., Pankow, T., Phan, E., Quataert, T., Quinn, S. W., Ruplin, C., Salem, D., Seitz, D. A., Sheppard, A., Siy, S. D., Summers, D., Szabo, A., Timofeeva, M., Vaivads, A., Velli, M., Yehle, A., Werthimer, D., and Wygant, J. R.: The FIELDS Instrument Suite for Solar Probe Plus: Measuring the Coronal Plasma and Magnetic Field, Plasma Waves and Turbulence, and Radio Signatures of Solar Transients, Space Sci. Rev., 204, 4982, https://doi.org/10.1007/s11214-016-0244-5, 2016.

Belheouane, S., Zaslavsky, A., Meyer-Vernet, N., Issautier, K., Mann, I., and Maksimovic, M.: Detection of Interstellar Dust with STEREO/WAVES at $1 \mathrm{AU}$, Sol. Phys., 281, 501-506, https://doi.org/10.1007/s11207-012-9995-7, 2012.

Burch, J., Moore, T., Torbert, R., and Giles, B.: Magnetospheric multiscale overview and science objectives, Space Sci. Rev., 199, 5-21, 2016.

Bougeret, J.-L., Kaiser, M. L., Kellogg, P. J., Manning, R., Goetz, K., Monson, S. J., Monge, N., Friel, L., Meetre, C. A., Perche, C., Sitruk, L., and Hoang, S.: WAVES: The radio and plasma wave investigation on the wind spacecraft, Space Sci Rev., 71, 231-263, https://doi.org/10.1007/BF00751331, 1995.
Calvinhac, L.: Dust fluxes in the inner heliosphere - Simulations of the conditions on the spacecraft Parker Solar Probe, Student project University Toulouse III, Sabatier, 2019.

Campanell, M. D.: Negative plasma potential relative to electron-emitting surfaces, Phys. Rev. E, 88, 033103-1-10, https://doi.org/10.1103/PhysRevE.88.033103, 2013.

Ceplecha, Z., Borovička, J., Elford, W. Gr., Revelle, D. O., Hawkes, R. L., Porubčan, V., and Šimek, M.: Meteor phenomena and bodies, Space Sci. Rev., 84, 327-471, https://doi.org/10.1023/A:1005069928850, 1998.

Collette, A., Gruün, E., Malaspina, D., and Sternovsky, Z.: Micrometeoroid impact charge yield for common spacecraft materials, J. Geophys. Res.-Space, 119, 6019-6026, https://doi.org/10.1002/2014JA020042, 2014.

Collette, A., Meyer, G., Malaspina, D., and Sternovsky, Z.: impact investigation of antenna signals from dust impacts on spacecraft, J. Geophys. Res., 120, 5298-5305, https://doi.org/10.1002/2015JA021198, 2015.

Collette, A., Malaspina, D. M., and Sternovsky, Z.: Characteristic temperatures of hypervelocity dust impact plasmas, J. Geophys. Res.-Space, 121, 2169-9402, https://doi.org/10.1002/2015JA022220, 2016.

Connors, M., Russell, C. T., and Lai, H. R.: A temporary earth co-orbital linked to interplanetary field enhancements, Mon. Notices Royal Astron. Soc., 443, L109-L113, https://doi.org/10.1093/mnrasl/slu092, 2014.

Czechowski, A. and Mann, I.: Collisional vaporization of dust and production of gas in the beta Pictoris dust disk, Astrophys. J., 660, 1541-1555, https://doi.org/10.1086/512965, 2007.

Czechowski, A. and Mann, I.: Formation and acceleration of nano dust in the inner heliosphere, Astrophys. J., 714, 89-99, https://doi.org/10.1088/0004-637X/714/1/89, 2010.

Czechowski, A. and Mann, I.: Nanodust in the Solar System: Discoveries and Interpretations, edited by: Mann, I., Meyer-Vernet, N., and Czechowski, A., Astrophysics and Space Science Library, Springer, New York, Vol. 385, 47-75, 2012.

Dietzel, H., Eichhorn, G., Fechtig, H., Grun, E., Hoffmann, H.-J., and Kissel, J.: The HEOS 2 and HELIOS micrometeoroid experiments, J. Phys. E, 6, 209-217, https://doi.org/10.1088/00223735/6/3/008, 1973.

Drapatz, S. and Michel, K. W.: Theory of shock-wave ionization upon high-velocity impact of micrometeorites. Zeitschrift für Naturforschung A, 29, 870-879, https://doi.org/10.1515/zna1974-0606, 1974.

Ergun, R. E., Malaspina, D. M., Bale, S. D., McFadden, J. P., Larson, D. E., Mozer, F. S., Meyer-Vernet, N., Maksimovic, M., Kellogg, P. J., and Wygant, J. R.: Spacecraft charging and ion wake formation in the near-Sun environment, Phys. Plasmas, 17, 072903, https://doi.org/10.1063/1.3457484, 2010.

Fox, N. J., Velli, M. C., Bale, S. D., Decker, R., Driesman, A., Howard, R. A., Kasper, J. C., Kinnison, J., Kusterer, M., Lario, D., Lockwood, M. K., McComas, D. J., Raouafi, N. E., and Szabo, A.: The Solar Probe Plus mission: humanity's first visit to our star, Space Sci. Rev., 204, 7-48, https://doi.org/10.1007/s11214-015-0211-6, 2015.

Göller, J. R. and Grün, E.: Calibration of the Galileo/Ulysses dust detectors with different projectile materials and at varying impact angles, Planet. Space Sci., 37, 1197-1206, https://doi.org/10.1016/0032-0633(89)90014-7, 1989. 
Gurnett, D. A.: Principles of Space Plasma Wave Instrument Design, Measurement Techniques for Space Plasmas, AGU Monograph 103, edited by: Pfaff, R., Borovsky, J., and Young, D., American Geophysical Union, Washington, DC, 121-136, 1998.

Gurnett, D. A., Huff, R. L., and Kirchner, D. L.: The wide-band plasma wave investigation, Space Sci. Rev., 79, 195-208, 1997a.

Gurnett, D. A., Ansher, J. A., Kurth, W. S., and Granroth, L. J.: Micron-sized dust particles detected in the outer solar system by the Voyager 1 and 2 plasma wave instruments, Geophys. Res. Lett., 24, 3125-3128, https://doi.org/10.1029/97GL03228, 1997b.

Gurnett, D. A., Kurth, W. S., Kirchner, D. L., Hospodarsky, G. B., Averkamp, T. F., Zarka, P., Lecacheux, A., Manning, R., Roux, A., Canu, P., Cornilleau-Wehrlin, N., Galopeau, P., Meyer, A., Bostrom, R., Gustafsson, G., Wahlund, J.-E., Aahlen, L., Rucker, H. O., Ladreiter, H.-P., Macher, W., Woolliscroft, L. J. C., Alleyne, H., Kaiser, M. L., Desch, M. D., Farrell, W. M., Harvey, C. C., Louarn, P., Kellogg, P. J., Goetz, K., and Pedersen, A.: The Cassini radio and plasma wave science investigation, Space Sci. Rev., 114, 395-463, https://doi.org/10.1007/s11214-004-1434-0, 2004.

Gustafsson, G., André, M., Carozzi, T., Eriksson, A. I., Fälthammar, C.-G., Grard, R., Holmgren, G., Holtet, J. A., Ivchenko, N., Karlsson, T., Khotyaintsev, Y., Klimov, S., Laakso, H., Lindqvist, P.-A., Lybekk, B., Marklund, G., Mozer, F., Mursula, K., Pedersen, A., Popielawska, B., Savin, S., Stasiewicz, K., Tanskanen, P., Vaivads, A., and Wahlund, J.-E.: First results of electric field and density observations by Cluster EFW based on initial months of operation, Ann. Geophys., 19, 1219-1240, 2001.

Grün, E., Pailer, N., Fechtig, H., and Kissel, J.: Orbital and physical characteristics of micrometeoroids in the inner solar system as observed by Helios 1, Planet. Space Sci., 28, 333-349, https://doi.org/10.1016/0032-0633(80)90022-7, 1980.

Grün, E., Zook, H. A., Fechtig, H., and Giese, R. H.: Collisional balance of the meteoritic complex, Icarus, 62, 244-272, https://doi.org/10.1016/0019-1035(85)90121-6, 1985.

Hornung, K. and Kissel, J.: On shock wave impact ionization of dust particles, Astron. Astrophys., 291, 324-336, 1994.

Ishimoto, H. and Mann, I.: Modeling the particle mass distribution within $1 \mathrm{AU}$ of the Sun, Planet. Space Sci. 47, 225-232, https://doi.org/10.1016/S0032-0633(98)00083-X, 1999.

Jakosky, B. M., Lin, R. P., Grebowsky, J. M., Luhmann, J. G., Mitchell, D. F., Beutelschies, G., Priser, T., Acuna, M., Andersson, L., Baird, D., Baker, D., Bartlett, R., Benna, M., Bougher, S., Brain, D., Carson, D., Cauffman, S., Chamberlin, P., Chaufray, J.-Y., Cheatom, O. Clarke, J., Connerney, J., Cravens, T., Curtis, D., Delory, G., Demcak, S., DeWolfe, A., Eparvier, F., Ergun, R., Eriksson, A., Espley, J., Fang, X., Folta, D., Fox, J., GomezRosa, C., Habenicht, S., Halekas, J., Holsclaw, G., Houghton, M., Howard, R., Jarosz, M., Jedrich, N., Johnson, M., Kasprzak, W., Kelley, M., King, T., Lankton, M., Larson, D., Leblanc, F., Lefevre, F., Lillis, R., Mahaffy, P., Mazelle, C., McClintock, W., McFadden, J., Mitchell, D. L., Montmessin, F., Morrissey, J., Peterson, W., Possel, W., Sauvaud, J. -A., Schneider, N., Sidney, W., Sparacino, S., Stewart, A. I. F., Tolson, R., Toublanc, D., Waters, C., Woods, T., Yelle, R., and Zurek, R.: The Mars atmosphere and volatile evolution (MAVEN) mission, Space Sci. Rev., 195, 3-48, 2015.
John, W., Reischl, G., and Devor, W.: Charge transfer to metal surfaces from bouncing aerosol particles, J. Aerosol Sci., 11, 115138, https://doi.org/10.1016/0021-8502(80)90029-4 1980.

Jones, A. P., Tielens, A. G. G. M., and Hollenbach, D. J.: Grain shattering in shocks: the interstellar grain size distribution, Astrophys. J., 469, 740-764, 1996.

Jones, G. H., Knight, M., Battams, K., Boice, D., Brown, J., Giordano, S., Raymond, J., Snodgrass, C., Steckloff, J., Weissman, P., Fitzsimmons, Al., Lisse, C., Opitom, C., Birkett, K., Bzowski, M., Decock, A., Mann, I., Ramanjooloo, Y., and McCauley, P.: The Science of Sungrazers, Sunskirters, and Other Near-Sun Comets, Space Sci. Rev., 214, 86 pp., 2018.

Juhasz, A. and Horanyi, M.: Dynamics and distribution of nanodust particles in the inner solar system, Geophys. Res. Lett., 40, 2500-2504, https://doi.org/10.1002/grl.50535, 2013.

Kasaba, Y., Bougeret, J.-L., Blomberg, L. G., Kojima, H., Yagitani, S., Moncuquet, M., Trotignon, J.-G., Chanteur, G., Kumamoto, A., Kasahara, Y., Lichtenberger, J., Omura, Y., Ishisaka, K., and Matsumoto, H.: The Plasma Wave Investigation (PWI) onboard the BepiColombo/ MMO: First measurement of electric fields, electromagnetic waves, and radio waves around Mercury, Planet. Space Sci., 58, 238-278, 2010.

Kellogg, P. J., Goetz, K., and Monson, S. J.: Dust impact signals on the wind spacecraft, J. Geophys. Res.-Space, 121, 966-991, https://doi.org/10.1002/2015JA021124, 2016.

Kellogg, P. J., Goetz, K., and Monson, S. J.: Are STEREO single hits dust impacts?, J. Geophys. Res.-Space, 123, 7211-7219, https://doi.org/10.1029/2018JA025554, 2018.

Kimura, H. Ishimoto, H., and Mukai, T.: A study on solar dust ring formation based on fractal dust models, Astron. Astrophys., 326, 263-270, 1997.

Lai, H. R., Wei, H. Y., and Russell, C. T.: Solar Wind Plasma Profiles During Interplanetary Field Enhancements (IFEs): Consistent with Charged-Dust Pickup, SOLAR WIND 13: AIP CP 1539, 402-405, https://doi.org/10.1063/1.4811070, 2013.

Lai, H. R., Russell, C. T., Jia, Y. D., Wei, H. Y., and Angelopoulos, V.: Momentum transfer from solarwind to interplanetary field enhancements inferred from magnetic field draping signatures, Geophys. Res. Lett., 42, 1640-1645, https://doi.org/10.1002/2015GL063302, 2015.

Le Chat, G., Zaslavsky, A., Meyer-Vernet, N., Issautier, K., Belheouane, S., Pantellini, F., Maksimovic, M., Zouganelis, I., Bale, S. D., and Kasper, J. C.: Effect of the Interplanetary Medium on Nanodust Observations by the Solar Terrestrial Relations Observatory, Sol. Phys., 286, 549-559, 2013.

Le Chat, G., Zaslavsky, A., Meyer-Vernet, N., Issautier, K., Belheouane, S., Pantellini, F., Maksimovic, M., Zouganelis, I., Bale, S. D., and Kasper, J. C.: Interplanetary nanodust detection by the Solar Terrestrial Relations Observatory/WAVES low frequency receiver, Sol. Phys., 290, 933-942, https://doi.org/10.1007/s11207-015-0651-x, 2015.

MacQueen, R. M.: Infrared observations of outer solar corona, Astrophys. J., 154, 1059, https://doi.org/10.1086/149825, 1968.

Maksimovic, M., Soucek, J., Bale, S. D., Bonnin, X., Chust, T., Khotyaintsev, Y., Plettermeier, D., Kretzschmar, M., Steller, M., and Štverák, Š.: The Radio and Plasma Waves (RPW) instrument on the Solar Orbiter Mission, Astron. Astrophys., submitted, 2019. 
Malaspina, D. M. and Wilson, L. B.: A database of interplanetary and interstellar dust detected by the Wind spacecraft, J. Geophys. Res.-Space, 121, 9369-9377, https://doi.org/10.1002/2016JA023209, 2016.

Malaspina, D. M., Horányi, M., Zaslavsky, A., Goetz, K., Wilson, L. B., and Kersten, K.: Interplanetary and interstellar dust observed by the Wind WAVES electric field instrument, Geophys. Res. Lett., 41, 266-272, https://doi.org/10.1002/2013GL058786, 2014.

Malaspina, D. M., O'Brien, L. E., Thayer, F., Sternovsky, Z., and Collette, A.: Revisiting STEREO interplanetary and interstellar dust flux and mass estimates, J. Geophys. Res.-Space, 120, 60856100, https://doi.org/10.1002/2015JA021352, 2015.

Mann, I.: The solar F-corona-Calculations of the optical and infrared brightness of circumsolar dust, Astron. Astrophys., 261, 329-335, 1992.

Mann, I.: Interstellar dust in the solar system, Ann. Rev. Astron. Astrophys. 48, 173-203, https://doi.org/10.1146/annurev-astro081309-130846, 2010.

Mann, I. and Czechowski, A.: Dust destruction and ion formation in the inner solar system, Astrophys. J., 621, L73-L76, https://doi.org/10.1086/429129, 2005.

Mann, I. and Murad, E.: On the existence of silicon nanodust near the Sun, Astrophys. J., 624, L125-L128, https://doi.org/10.1086/430701, 2005.

Mann, I., Kimura, H., Biesecker, D., Tsurutani, B., Grün, E., McKibben, R. B., Liou, J-C., MacQueen, R., Mukai, T., Guhathakurta, M., and Lamy, P.: Dust near the Sun, Space Sci. Rev., 110, 269305, https://doi.org/10.1023/B:SPAC.0000023440.82735.ba, 2004.

Mann, I., Czechowski, A., and Meyer-Vernet, N.: Dust in the interplanetary medium - interactions with the solar wind, Solar Wind 12, AIP CP 1216, 491-496, https://doi.org/10.1063/1.3395911, 2010.

Mann, I., Pellinen-Wannberg, A., Murad, E., Popova, O., MeyerVernet, N.,Rosenberg, M., Mukai, T., Czechowski, A., Mukai, S., Safrankova, J., and Nemecek, Z.: Dusty Plasma Effects in Near Earth Space and Interplanetary Medium, Space Sci. Rev., 161, 1-47, https://doi.org/10.1007/s11214-011-9762-3, 2011.

Mann, I., Meyer-Vernet, N., and Czechowski, A.: Dust in the planetary system: Dust interactions in space plasmas of the solar system, Phys. Rep., 536, 1-39, https://doi.org/10.1016/j.physrep.2013.11.001, 2014.

Mann, I., Antonsen, T., Nouzak, L., and Vaverka, J.: Replication Data for: Dust observations with antenna measurements and its prospects for observations with Parker Solar Probe and Solar Orbiter, DataverseNO, https://doi.org/10.18710/LURI1R, last access: 4 December 2019.

McBride, N. and McDonnell, J. A. M.: Meteoroid impacts on spacecraft: Sporadics, streams, and the 1999 Leonids, Planet. Space Sci., 47, 1005-1013, https://doi.org/10.1016/S00320633(99)00023-9, 1999.

McCrea, I., Aikio, A., Alfonsi, L., Belova, E., Buchert, S., Clilverd, M., Engler, N., Gustavsson, B., Heinselman, Cr., Kero, J., Kosch, M., Lamy, H., Leyser, T., Ogawa, Y., Oksavik, K., PellinenWannberg, A., Pitout, F., Rapp, M., Stanislawska, I., and Vierinen, J.: The science case for the EISCAT_3D radar, Prog. Earth Planet. Sci., 2, 1-63, https://doi.org/10.1186/s40645-015-0051$8,2015$.
McDonnell, J. A. M. and Gardner, D. J.: Meteoroid morphology and densities: Decoding satellite impact data, Icarus, 133, 25-35, https://doi.org/10.1006/icar.1998.5912, 1998.

Meyer-Vernet, N.: Comet Giacobini-Zinner diagnosis from radio measurements, Adv. Space Res., 5, 37-46, 1985.

Meyer-Vernet, N.: Detecting dust with electric sensors in planetary rings, comets and interplanetary space, ESA SP-476, 635-639, 2001.

Meyer-Vernet, N., Maksimovic, M., Czechowski, A., Mann, I., Zouganelis, I., Goetz, K., Kaiser, M. L., St. Cyr, O. C., Bougeret, J.-L., and Bale, S. D.: Dust Detection by the Wave Instrument on STEREO: Nanoparticles Picked up by the Solar Wind, Sol. Phys., 256, 463-474, https://doi.org/10.1007/s11207-009-93492, 2009.

Meyer-Vernet, N., Moncuquet, M., Issautier, K., and Lecacheux, A.: The importance of monopole antennas for dust observations: why Wind/WAVES does not detect nanodust, Geophys. Res. Lett., 41, 2716-2720, https://doi.org/10.1002/2014GL059988, 2014.

Meyer-Vernet, N., Moncuquet, M., Issautier, K., and Schippers, P.: Frequency range of dust detection in space with radio and plasma wave receivers: theory and application to interplanetary nanodust impacts on Cassini, J. Geophys. Res., 121, 8-22, https://doi.org/10.1002/2016JA023081, 2017.

Minato, T., Köhler, M., Kimura, H., Mann, I., and Yamamoto, T.: Momentum transfer to interplanetary dust from the solar wind, Astron. Astrophys., 424, L13-L16, https://doi.org/10.1051/0004-6361:200400037, 2004.

Miyachi, T., Fujii, M., Hasebe, N., Miyajima, M., Okudaira, O., Takechi, S., Onishi, T., Minami, S., Shibata, H., Ohashi, H., and Iwai, T.: Measurement of temperature after hypervelocity collision of microparticles in the range from 10 to $40 \mathrm{~km} \mathrm{~s}^{-1}$, Appl. Phys. Lett., 93, 174107, https://doi.org/10.1063/1.3013313, 2008.

Mocker, A., Bugiel, S., Auer, S.,Baust, G., Colette, A., Drake, K., Fiege, K., Grün, E., Heckmann, F., Helfert, S.,Hillier, J., Kempf, S, Matt, G, Mellert, T., Munsat, T, Otto, K., Postberg, F., Shu, A., Sternovsky, Z., and Srama, R.: A 2 MV Van de Graaff accelerator as a tool for planetary and impact physics research, Rev. Sci. Instrum., 82, 095111, https://doi.org/10.1063/1.3637461, 2011.

Mueller, M.: The SOlar Orbiter Mission, Astron. Astrophys., submitted, 2019.

Mukai, T. and Mukai, S.: Temperature and motion of grains in interplanetary space, Publ. Astronom. Soc. Jpn., 25, 481-488, 1973.

Mukai, T. and Yamamoto, T.: Model of the circumsolar dust, Publ. Astronom. Soc. Jpn., 31, 585-596, 1979.

Němeček, Z., Pavlů, J., Šafránková, J., Beránek, M., Richterová, I., Vaverka, J., and Mann, I.: Lunar Dust Grain Charging by Electron Impact: Dependence of the Surface Potential on the Grain Size, Astrophys. J., 738, 1-7, https://doi.org/10.1088/0004637X/738/1/14, 2011.

Nesvorný, D., Janches, D., Vokrouhlický, D., Pokorny, P., Bottke, W. F., and Jenniskens, P.: Dynamical Model for the Zodiacal Cloud and Sporadic Meteors, Astrophys. J., 743, 1-16, 2011.

Nogami, K., Fujii, M., Ohashi, H., Miyachi, T., Sasaki, S., Hasegawa, S., Yano, H., Shibata, H., Iwai, T., Minami, S., Takechi, S., Grün, E., and Srama, R.: Development of the Mercury dust monitor (MDM) onboard the BepiColombo mission, Planet. Space Sci., 58, 108-115, 2010. 
Nouzák, L., Hsu, S., Malaspina, D., Thayer, F. M., Ye, S. Y., Pavlů, J., Němeček, Z., Šafránková, J., and Sternovsky, Z.: Laboratory modeling of dust impact detection by the Cassini spacecraft, Planet. Space Sci., 156, 85-91, https://doi.org/10.1016/j.pss.2017.11.014, 2018.

Ohgaito, R., Mann, I., Kuhn, J. R., MacQueen, R. M., and Kimura, H.: The J- and K-band brightness of the solar F corona observed during the solar eclipse on 1998 February 26, Astrophys. J., 578, 610-620, https://doi.org/10.1086/342426, 2002.

Pantellini, F., Belheouane, S., Meyer-Vernet, N., and Zaslavsky, A.: Nano dust impacts on spacecraft and boom antenna charging, Astrophys. Space Sci., 341, 309-314, https://doi.org/10.1007/s10509-012-1108-4, 2012.

Pellinen-Wannberg, A., Kero, J., Häggström, I., Mann, I., and Tjulin, A.: The forthcoming EISCAT_3D as an extraterrestrial matter monitor, Planet. Space Sci., 123, 33-40, https://doi.org/10.1016/j.pss.2015.10.009, 2016.

Plane, J. M. C.: Cosmic dust in the earth's atmosphere, Chem. Soc. Rev., 41, 6507-6518, https://doi.org/10.1039/c2cs35132c, 2012.

Ragot, B. R. and Kahler, S. W.: Interactions of dust grains with coronal mass ejections and solar cycle variations of the F-coronal brightness, Astrophys. J., 594, 1049-1059, https://doi.org/10.1086/377076, 2003.

Russell, C. T., Luhmann, J., Barnes, A., Mihalov, J. D., and Elphic, R. C.: An unusual interplanetary event - encounter with a comet, Nature, 305, 612-615, https://doi.org/10.1038/305612a0, 1983.

Sánchez-Lavega, A., García Muñoz, A., García-Melendo, E., PérezHoyos, S., Gómez-Forrellad, J. M., Pellier, C., Delcroix, M., López-Valverde, M. A., González-Galindo, F., Jaeschke, W., Parker, D., Phillips, J., and Peach, D.: An extremely high-altitude plume seen at Mars' morning terminator, Nature, 518, 525-528, https://doi.org/10.1038/nature14162, 2015.

Schippers, P., Meyer-Vernet, N., Lecacheux, A., Kurth, W. S., Mitchell, D. G., and André, N.: Nanodust detection near $1 \mathrm{AU}$ from spectral analysis of Cassini/Radio and Plasma Wave Science data, Geophys. Res. Lett., 41, 5382-5388, https://doi.org/10.1002/2014GL060566, 2014.

Schippers, P., Meyer-Vernet, N., Lecacheux, A., Belheouane, S., Moncuquet, M., Kurth, W. S., and Mann, I.: Nanodust detection between 1 and $5 \mathrm{AU}$ using Cassini wave measurements, Astrophys. J., 806, 1-7, https://doi.org/10.1088/0004-637X/806/1/77, 2015.

Schultz, P. H., Sugita, S., Eberhardy, C. A., and Ernst, C. M.: The role of ricochet impacts on impact vaporization. International journal of impact engineering, 33, 771-780, https://doi.org/10.1016/j.ijimpeng.2006.09.005, 2006.

Shu, A., Collette, A., Drake, K., Grün, E., Horanyi, M., Kempf, S., Mocker, A., Munsat, T., Northway, P., Srama, R., Sternovsky, Z., and Thomas, E.: $3 \mathrm{MV}$ hypervelocity dust accelerator at the Colorado center for lunar dust and atmospheric studies, Rev. Sci. Instrum., 83, 5108, https://doi.org/10.1063/1.4732820, 2012.

Srama, R., Ahrens, T. J., Altobelli, N., Auer, S., Bradley, J. G., Burton, M., Dikarev, V. V., Economou, T., Fechtig, H., Görlich, M., Grande, M., Graps, A., Grün, E., Havnes, O., Helfert, S., Horanyi, M., Igenbergs, E., Jessberger, E. K., Johnson, T. V., Kempf, S. Krivov, A. V., Krüger, H., Mocker-Ahlreep, A., MoragasKlostermeyer, G., Lamy, P., Landgraf, M., Linkert, D., Linkert, G., Lura, F., McDonnell, J. A. M., Möhlmann, D., Morfill, G. E., Müller, M., Roy, M., Schäfer, G., Schlotzhauer, G., Schwehm,
G. H., Spahn, F., Stübig, M., Svestka, J., Tschernjawski, V., Tuzzolino, A. J., Wäsch, R., and Zook, H. A.: The Cassini cosmic dust analyzer, in: The Cassini-Huygens Mission, Springer, Dordrecht, 465-518, https://doi.org/10.1007/978-1-4020-2774-1_7, 2004.

Stauffer, J. R., Stenborg, G., and Howard, R. A.: Measuring the Flattening of the Outer F-corona Using STEREO-A/HI-1 Images, Astrophys. J., 864, 1-10, https://doi.org/10.3847/15384357/aad689, 2018.

Stenborg, G. and Howard, R. A.: A Heuristic Approach to Remove the Background Intensity on White-light Solar Images, I. STEREO/HI-1 Heliospheric Images, Astrophys. J., 839, 1-16, https://doi.org/10.3847/1538-4357/aa6a12, 2017a.

Stenborg, G. and Howard, R. A.: The Evolution of the Surface of Symmetry of the Interplanetary Dust from $24^{\circ}$ to $5^{\circ}$ Elongation, Astrophys. J., 848, 1-13, https://doi.org/10.3847/15384357/aa8ef0, 2017b.

Stenborg, G., Howard, R. A., and Stauffer, J. R.: Characterization of the White-light Brightness of the F-corona between $5^{\circ}$ and $24^{\circ}$ Elongation, Astrophys. J., 862, 1-21, https://doi.org/10.3847/1538-4357/aacea3, 2018.

Thayer, F. M., Malaspina, D. M., Collette, A., and Sternovsky, Z.: Variation in relative dust impact charge recollection with antenna to spacecraft potential on STEREO, J. Geophys. Res.-Space, 121, 4998-5004, https://doi.org/10.1002/2015JA021983, 2016.

Torbert, R. B., Russell, C. T., Magnes, W., Ergun, R. E., Lindqvist, P.-A., Le Contel, O., Vaith, H., Macri, J., Myers, S., Rau, D., Needell, J., King, B., Granoff, M., Chutter, M., Dors, I., Olsson, G., Khotyaintsev, Y. V., Eriksson, A., Kletzing, C. A., Bounds, S. Anderson, B., Baumjohann, W., Steller, M., Bromund, K., Le, Guan, Nakamura, R., Strangeway, R. J., Leinweber, H. K., Tucker, S., Westfall, J., Fischer, D., Plaschke, F., Porter, J., and Lappalainen, K.: The FIELDS instrument suite on MMS: Scientific objectives, measurements, and data products, Space Sci. Rev., 199, 105-135, 2016.

Tsintikidis, D., Gurnett, D. A., Granroth, L. J., Allendorf, S. C., and Kurth, W. S.: A revised analysis of micron-sized particle detected near Saturn by the Voyager 2 plasma wave instrument, J. Geophys. Res., 99, 2261, https://doi.org/10.1029/93JA02906, 1994.

Vaverka, J., Pellinen-Wannberg, A., Kero, J., Mann, I., De Spiegeleer, A., Hamrin, M., Norberg, C., and Pitkänen, T.: Detection of meteoroid hypervelocity impacts on the Cluster spacecraft: First results, J. Geophys. Res.-Space, 122, 6485-6494, https://doi.org/10.1002/2016JA023755, 2017a.

Vaverka, J., Pellinen-Wannberg, A., Kero, J; Mann, I., De Spiegeleer, A., Hamrin, M., Norberg, C., and Pitkänen, T.: Potential of Earth Orbiting Spacecraft Influenced by Meteoroid Hypervelocity Impacts, IEEE Trans. Plasma Sci., 45, 2048-2055, https://doi.org/10.1109/TPS.2017.2676984, 2017b.

Vaverka, J., Nakamura, T., Kero, J., Mann, I., De Spiegeleer, A., Hamrin, M., Norberg, C., Lindqvist, P-A., and PellinenWannberg, A.: Comparison of Dust Impact and Solitary Wave Signatures Detected by Multiple Electric Field Antennas Onboard the MMS Spacecraft, J. Geophys. Res.-Space, 123, 61196129, https://doi.org/10.1029/2018JA025380, 2018.

Wehry, A. and Mann, I.: Identification of beta-meteoroids from measurements of the dust detector onboard the Ulysses spacecraft, Astron. Astrophys., 341, 296-303, 1999. 
Wood, S. R., Malaspina, D. M., Andersson, L., and Horanyi, M.: Hypervelocity dust impacts on the Wind spacecraft: Correlations between Ulysses and Wind interstellar dust detections, J. Geophys. Res.-Space, 120, 7121-7129, https://doi.org/10.1002/2015JA021463, 2015.

Ye, S. Y., Gurnett, D. A., Kurth, W. S., Averkamp, T. F., Morooka, M., Sakai, S., and Wahlund, J. E.: Electron density inside Enceladus plume inferred from plasma oscillations excited by dust impacts, J. Geophys. Res.-Space, 119, 3373-3380, https://doi.org/10.1002/2014JA019861, 2014.

Ye, S. Y., Gurnett, D. A., and Kurth, W. S.: In-situ measurements of Saturn's dusty rings based on dust impact signals detected by Cassini RPWS, Icarus, 279, 51-61, https://doi.org/10.1016/j.icarus.2016.05.006, 2016a.

Ye, S.-Y., Kurth, W. S., Hospodarsky, G. B., Averkamp, T. F., and Gurnett, D. A.: Dust detection in space using the monopole and dipole electric field antennas, J. Geophys. Res.-Space, 121, 11964-11972, https://doi.org/10.1002/2016JA023266, 2016 b.

Ye, S.-Y., Kurth, W. S., Hospodarsky, G. B., Persoon, A. M., Gurnett, D. A., Morooka, M., Wahlund, J.-E., Hsu, H.-W., Seiss, M., and Srama, R.: Cassini RPWS dust observation near Janus and Epimetheus orbits, J. Geophys. Res., 123, 4952-4960, https://doi.org/10.1029/2017JA025112, 2018a.
Ye, S. Y., Kurth, W. S., Hospodarsky, G. B., Persoon, A. M., Sulaiman, A. H., Gurnett, D. A., Morooka, M., Wahlund, J. E., Hsu, H. W., Sternovsky, Z., and Wang, X.: Dust Observations by the Radio and Plasma Wave Science Instrument During Cassini's Grand Finale, Geophys. Res. Lett., 45, 10-101, 2018 b.

Ye, S.-Y., Vaverka, J., Nouzak, L., Sternovsky, Z., Zaslavsky, A., Pavlu, J., Mann, I., Hsu, H.-W., Averkamp, T. F., Sulaiman, A. H., Pisa, D., Hospodarsky, G. B., Kurth, W. S., and Horanyi, M.: Understanding Cassini RPWS Antenna Signals Triggered by Dust Impacts (supporting information), Geophys. Res. Lett., 46, 10941-10950, https://doi.org/10.1029/2019GL084150, 2019.

Zaslavsky, A.: Floating potential perturbations due to micrometeoroid impacts: Theory and application to S/WAVES data, J. Geophys. Res., 120, 855-867, https://doi.org/10.1002/2014JA020635, 2015.

Zaslavsky, A., Meyer-Vernet, N., Mann, I., Czechowski, A., Issautier, K., Le Chat, G., Pantellini, F., Goetz, K., Maksimovic, M., Bale, S. D., and Kasper, J. C.: Interplanetary dust detection by radio antennas: Mass calibration and fluxes measured by STEREO/WAVES, J. Geophys. Res., 117, A05102, https://doi.org/10.1029/2011JA017480, 2012. 